Geometry $\&$ Topology

Volume 8 (2004) 1243-1280

Published: 24 September 2004

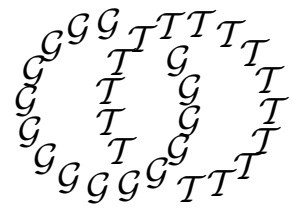

\title{
Cylindrical contact homology of subcritical Stein-fillable contact manifolds
}

\author{
MEI-Lin YAU \\ Department of Mathematics, Michigan State University \\ East Lansing, MI 48824, USA \\ Email: yau@math.msu.edu
}

\begin{abstract}
We use contact handle decompositions and a stabilization process to compute the cylindrical contact homology of a subcritical Stein-fillable contact manifold with vanishing first Chern class, and show that it is completely determined by the homology of a subcritical Stein-filling of the contact manifold.
\end{abstract}

\section{AMS Classification numbers Primary: 57R17}

Secondary: 57R65, 53D40, 58C10

Keywords: Subcritical Stein-fillable contact manifold, cylindrical contact homology, holomorphic curves, contact handles, Reeb vector field

Proposed: Yasha Eliashberg

Seconded: Leonid Polterovich, Ronald Fintushel
Received: 13 March 2004

Revised: 2 July 2004 


\section{Introduction}

A 1 -form $\alpha$ on a $(2 n-1)$-dimensional oriented manifold $M$ is called a contact 1 -form if it satisfies the contact condition:

$$
\alpha \wedge(d \alpha)^{n-1} \neq 0 \text { everywhere. }
$$

Its kernel $\xi=\{\alpha=0\}$ is called a (co-orientable) contact structure. $\xi$ is a codimension 1 tangent distribution with maximal non-integrability. The pair $(M, \xi)$ is called a contact manifold. Sometimes we write $(M, \alpha)$ to stress the contact $1-$ form $\alpha$ instead of the contact structure defined by $\alpha$. Note that if $\alpha$ is a contact 1 -form then so is $f \alpha$ for any $f \in C^{\infty}\left(M, \mathbb{R}_{+}\right)$, and $\operatorname{ker}(\alpha)=\operatorname{ker}(f \alpha)$. In this paper we assume $\xi=\operatorname{ker} \alpha$ to be positive, ie, $\alpha \wedge(d \alpha)^{n-1}>0$ is a volume form of $M$. Two contact manifolds $(M, \xi)$ and $\left(M^{\prime}, \xi^{\prime}\right)$ are contactomorphic if there is a diffeomorphism $\phi: M \rightarrow M^{\prime}$ such that $\phi_{*} \xi=\xi^{\prime} . \phi$ is called a contactomorphism. Contact manifolds, which include many $S^{1}$-bundles and hypersurfaces of symplectic manifolds, and eventually every 3 -manifold, were first introduced in [25] and [26], and has been under study for decades.

By the contact version of Darboux's theorem, all contact 1-forms are locally isomorphic, which implies that there is no local invariant for a contact structure. Moreover, it is proved by Gray in [16 that if two contact structures on a closed contact manifold are homotopic as contact structures, then they are isotopic as contact structures. Therefore there are also no local invariants of the space of contact structures on a closed manifold. Note that the contact condition (1) implies that $d \alpha$ restricts to a symplectic structure on $\xi$. The conformal class of such symplectic structures is independent of the choice of a defining contact 1 -form for $\xi$. Thus we can endow $\xi$ with a $d \alpha$-compatible almost complex structure and the first Chern class $c_{1}(\xi)$ is an invariant of $\xi$.

On the other hand, there are many contact structures which are homotopic as hyperplane distributions (hence have the same $c_{1}(\xi)$ ) but not homotopic as contact structures $([15,22],[23$, 32], 33, etc). This fact makes the classification of contact structures an interesting and challenging problem. For contact 3-manifolds, many nice partial results have been obtained (7], 15], 22], 23], [24]). But much less is known for higher dimensional cases (13], 32], 33]).

Contact Homology Theory (10, see also [33, 1], 2]), introduced by Y Eliashberg and H Hofer in 1994 and has been expanded into a bigger framework Symplectic Field Theory (12], 3]) provides Floer-Gromov-Witten type of invariants to distinguish non-isomorphic contact structures on closed manifolds: A contact 1 -form $\alpha$ of $M$ associates a unique vector field $R_{\alpha}$ which satisfies

$$
\alpha\left(R_{\alpha}\right)=1, \quad d \alpha\left(R_{\alpha}, \cdot\right)=0 .
$$


$R_{\alpha}$ is called the Reeb vector field (of $\left.\alpha\right) .(M, \xi:=\operatorname{ker} \alpha$ ) also associates a symplectic manifold $\left(\mathbb{R} \times M, d\left(e^{t} \alpha\right)\right)$, the symplectization of $(M, \xi)$, whose symplectic structure $d\left(e^{t} \alpha\right)$ depends (up to an $\mathbb{R}$-invariant diffeomorphism of $\mathbb{R} \times M$ ) only on $\xi$. Then contact homology of $(M, \xi)$ is defined by suitably counting in $\mathbb{R} \times M(1+s)$-punctured pseudo-holomorphic spheres which converges exponentially to good periodic Reeb trajectories at $t= \pm \infty$ at punctures. In some favorable cases (see section 2) one can count only pseudo-holomorphic cylinders connecting good contractible Reeb orbits and define cylindrical contact homology $H C(M, \xi)$ of $(M, \xi)$. In this paper we consider only the $c_{1}(\xi)=0$ case, then $H C(M, \xi)$ is graded by the reduced Conley-Zehnder index of Reeb orbits. The construction of $H C(M, \xi)$ involves choices of a contact 1-form $\alpha$ and an $\alpha$-admissible almost complex structure. Yet the resulting contact homology is independent of all these extra choices and is truly an invariant of isotopy classes of contact structures. Though the full strength of contact homology is yet to be explored, some interesting classification results have been obtained in the spirit of (cylindrical) contact homology theory (4], 32, 33, see also [12, 1]).

Though contact homology is meant to distinguish non-isomorphic contact structures, itself is actually an subject of interest. One would like to know what contact homology tells about a contact manifold. Thus it is important to compute some concrete examples and develop computational mechanisms of contact homology.

This paper focuses on the computation of cylindrical contact homology of subcritical Stein-fillable contact manifolds. A complex $n$-dimensional Stein domain $(V, J)$ is called subcritical if it admits a proper, strictly $J$-convex Morse function with finitely many critical points and all critical points have Morse index $<n$. Such a function is called subcritical. A contact manifold is called subcritical Stein-fillable if it is the boundary of some subcritical Stein domain and its contact structure is the corresponding CR-structure, ie, the field of maximal complex tangencies. Equivalently a subcritical Stein-fillable $(M, \xi)$ can be identified with a regular level set of a subcritical strictly $J$-convex function on a Stein manifold. From now on we will often use the shorthand " $S S F C$ " for "subcritical Stein-fillable contact" and simply call a subcritical Stein-fillable contact manifold a SSFC manifold, and similarly call a Stein-fillable contact manifold a $S F C$ manifold. In this paper we obtain the following result.

Main Theorem Let $(M, \xi)$ be a $(2 n-1)$-dimensional SSFC manifold with $n \geq 2,\left.c_{1}(\xi)\right|_{\pi_{2}(M)}=0$, and $(V, J)$ a subcritical Stein domain such that $\partial V=$ $M$ and $\xi$ is the maximal complex subbundle of $T M$. Then

$$
H C_{i}(M, \xi) \cong \underset{m \in \mathbb{N} \cup\{0\}}{\oplus} H_{2(n+m-1)-i}(V) .
$$


The Main Theorem results from the fact that, roughly speaking, counting pseudo-holomorphic cylinders is equivalent to counting gradient trajectories that connect critical points of consecutive indexes of a Morse function of a Stein filling of $(M, \xi)$. Hence the theorem shows that the contact homology of a SSFC manifold $(M, \xi)$ recovers in a way the homology of a Stein domain bounded by $M$.

Here is a brief outline of this paper: After introducing cylindrical contact homology in Section 2 we study in section 3 Reeb dynamics on subcritical contact handles, the building block of SSFC manifolds. Global dynamics on $M$ is discussed in Section 4. It is shown there that, since $(M, \xi)$ is subcritical, one gets enough room to maneuver attaching handles and hence contact 1-forms to show that contact homology of $(M, \xi)$ is essentially generated by Reeb orbits contained in cocores of contact handles. To compute $H C(M, \xi)$ we introduce in Section $5\left(M^{\prime}, \xi^{\prime}\right)$, the stabilization of $(M, \xi) .\left(M^{\prime}, \xi^{\prime}\right)$ is a SSFC manifold containing $(M, \xi)$ as a codimension 2 contact submanifold with $M^{\prime} \backslash M \cong V \times S^{1}$ a trivial $S^{1}$-bundle over a Stein-filling $V$ of $M$. By shaping contact handles of $\left(M^{\prime}, \xi^{\prime}\right)$ one finds that cylindrical contact homologies of $(M, \xi)$ and $\left(M^{\prime}, \xi^{\prime}\right)$ can be represented by the same set of generators with degrees shifted by 2 . In Section [6] we prove $H C_{*}(M, \xi) \cong H C_{*+2}\left(M^{\prime}, \xi^{\prime}\right)$. In Section 7 we prove that the counting of pseudo-holomorphic cylinders in $\left(M^{\prime}, \xi^{\prime}\right)$ is equivalent to the counting of gradient trajectories in a subcritical Stein-filling $V$ of $M$ and hence deduce the Main Theorem. To this end we first show that for generic $S^{1}$ invariant admissible almost complex structure the linearized $\bar{\partial}$-operator at an $S^{1}$-invariant solution is surjective. This is done by identifying it with the corresponding surjectivity problem in Floer Theory. Then by applying branched covering maps on $M^{\prime}$ and the said surjectivity result to show that up to contact isotopies there are only $S^{1}$-invariant solutions to be counted.

\section{Cylindrical contact homology}

Before introducing the cylindrical contact homology we would like to give a brief account on the reduced Conley-Zehnder index of a contractible Reeb orbit at first.

Let $S p(2 n)=S p(2 n, \mathbb{R})$ denote the group of symplectic $2 n \times 2 n$-matrices. For a path $\Phi:[0,1] \rightarrow S p(2 n)$ a Conley-Zehnder index (also called $\mu$-index) $\mu(\Phi)$ is defined in terms of crossing numbers (30]). Here we refer readers to [30] for a precise definition of $\mu$ and to $[6$ for the original definition and general properties of $\mu$. We point out here that if $\Phi^{\prime}$ is a path in $S p\left(2 n^{\prime}\right)$ and $\Phi^{\prime \prime}$ is 
a path in $S p\left(2 n^{\prime \prime}\right)$ then $\mu\left(\Phi^{\prime} \oplus \Phi^{\prime \prime}\right)=\mu\left(\Phi^{\prime}\right)+\mu\left(\Phi^{\prime \prime}\right)$, here $S p\left(2 n^{\prime}\right) \oplus S p\left(2 n^{\prime \prime}\right)$ is identified as a subgroup of $S p\left(2 n^{\prime}+2 n^{\prime \prime}\right)$ in the obvious way. The following example shows that when $n=1, \mu / 2$ is roughly the winding number of $\Phi$.

Example Fix $T>0$ and $A \in \operatorname{sp}(2)=s l(2)$. Consider the path $\gamma:[0, T] \rightarrow$ $e^{t A} \in S p(2)$. Then

- $\mu(\gamma)=0$ if $A=\left(\begin{array}{ll}0 & b \\ a & 0\end{array}\right)$ for some constants $a>0, b>0$

- if $A=\left(\begin{array}{cc}0 & -1 \\ 1 & 0\end{array}\right)$ then $\mu(\gamma)=m, m=2 n+1$ if $n \pi<T<(n+1) \pi$, $m=2 n$ if $T=n \pi$.

For computational convenience we define the reduced Conley-Zehnder index (also called $\bar{\mu}$-index) of a path $\Phi$ in $S p(2 n-2)$ to be

$$
\bar{\mu}(\Phi)=\mu(\Phi)+(n-3) .
$$

Fix a contact 1 -form $\alpha$ on a $(2 n-1)$-dimensional contact manifold $(M, \xi)$. Let $\gamma:[o, \tau] \rightarrow M$ be a Reeb trajectory with $\dot{\gamma}(t)=R_{\alpha}(\gamma(t))$. Define the action $\mathcal{A}(\gamma)$ of $\gamma$ to be the number

$$
T=\mathcal{A}(\gamma):=\int_{\gamma} \alpha
$$

The flow $\left(R_{\alpha}\right)^{t}$ of $R_{\alpha}$ preserves $\xi$. Thus the linearized Reeb flow $\left(R_{\alpha}\right)_{*}^{t}$, when restricted on $\gamma$, defines a path of symplectic maps

$$
\Upsilon(t)=\left(R_{\alpha}\right)_{*}^{t}(\gamma(0)):\left.\left.\xi\right|_{\gamma(0)} \rightarrow \xi\right|_{\gamma(t)} .
$$

When $\gamma$ is periodic with period $T, \Upsilon(T)$ is called the linearized Poincaré return map along $\gamma$. We call $\gamma$ non-degenerate if 1 is not an eigenvalue of $\Upsilon(T)$, simple if $\gamma$ is not a nontrivial multiple cover of another Reeb orbit. A contact 1 -form $\alpha$ is called regular if every (contractible) Reeb orbit of $\alpha$ is non-degenerate. It is well-known that generic contact 1 -forms are regular. If we identify $\xi_{\gamma(T)}$ with $\mathbb{R}^{2 n-2}$ then $\Upsilon(T) \in S p(2 n-2)$ is a symplectic matrix. The eigenvalues of a symplectic matrix comes in pairs $\rho, \rho^{-1}$.

Assume $\gamma$ is a contractible periodic Reeb trajectory with action $T$. Let $D$ be a spanning disc of $\gamma$ and $\Psi:\left.\xi\right|_{D} \rightarrow \mathbb{R}^{2 n-2} \times D$ a symplectic trivialization of $\xi$ over $D$. Then $(\gamma, \Phi)$ defines a path $\left.\left(\Psi \circ \Upsilon \circ \Psi^{-1}\right)\right|_{\gamma}:[0, T] \rightarrow S p(2 n-2)$ starting from $I d$. The $\mu$-index of $(\gamma, D)$ is defined to be

$$
\mu(\gamma, \xi, D):=\mu\left(\left.\Psi \circ \Upsilon \circ \Psi^{-1}\right|_{\gamma}\right),
$$


and the corresponding $\bar{\mu}$-index is

$$
\bar{\mu}(\gamma, \xi, D)=\mu(\gamma, \xi, D)+(n-3) .
$$

Since $D$ is contractible, $\bar{\mu}(\gamma, \xi, D)$ does not depend on $\Psi$. Let $D^{\prime}$ be another spanning disc of $\gamma$. Then

$$
\bar{\mu}(\gamma, \xi, D)-\bar{\mu}\left(\gamma, \xi, D^{\prime}\right)=2 c_{1}(A)
$$

where $c_{1}(A):=c_{1}(\xi)(A), c_{1}(\xi)$ is the first Chern class of $\xi$ and $A=[D \cup$ $\left.D^{\prime}\right] \in H_{2}(M, \mathbb{Z})$. In this paper we will only consider $c_{1}(\xi)=0$ case, therefore $\bar{\mu}(\gamma, \xi)=\bar{\mu}(\gamma, \xi, D)$ is independent of the choice of a spanning disc and is denoted as $\bar{\gamma}$ for notational simplicity.

For a Reeb orbit $\gamma$ we denote by $\gamma^{m}$ the $m$-th multiple of $\gamma$. Recall $\Upsilon(T)$ the Poincaré return map of $\gamma$. Let $n(\gamma)$ denote the number of real negative eigenvalues of $\Upsilon(T)$ from the interval $(-1,0) . n(\gamma)$ does not depend on the trivialization of $\xi_{\gamma(T)}$.

Definition 2.1 A Reeb orbit $\sigma$ is said to be good if

$$
\sigma \neq \gamma^{2 m} \quad \text { for any } \gamma \text { with } n(\gamma)=\text { odd, } m \in \mathbb{N} \text {. }
$$

For the rest of the paper we will use the notation $\mathcal{P}=\mathcal{P}(\alpha)$ to denote the set of all good contractible Reeb orbits of $\alpha$. Good contractible Reeb orbits with any positive multiplicity are included in $\mathcal{P}$ as individual elements. Those contractible orbits not included in $\mathcal{P}$ are called bad. The exclusion of these bad orbits is necessary in order to define coherent orientations of moduli spaces of pseudo-holomorphic curves (see section 1.9 of [12] for more detail).

We now consider a class of almost complex structures on the symplectization

$$
\operatorname{Symp}(M, \alpha):=\left(\mathbb{R} \times M, d\left(e^{t} \alpha\right)\right)
$$

of $(M, \xi=\operatorname{ker} \alpha)$. An almost complex structure $J: \xi \rightarrow \xi$ on $\xi$ is called $d \alpha$-compatible if

$$
\begin{aligned}
d \alpha(x, J x) & >0 \text { for nonzero } x \in \xi, \\
d \alpha(J x, J y) & =d \alpha(x, y) \text { for } x, y \in \xi .
\end{aligned}
$$

This compatibility property dose not depend on the choice of $\alpha$. Note that $d \alpha(\cdot, J \cdot)$ is a Riemannian metric on $\xi$. A $d \alpha$-compatible $J$ can be extended uniquely to a $d\left(e^{t} \alpha\right)$-compatible almost complex structure on $\operatorname{Symp}(M, \alpha)$, also denoted by $J$ by the abuse of language, such that

$$
J\left(\frac{\partial}{\partial t}\right)=R_{\alpha}, \quad J\left(R_{\alpha}\right)=-\frac{\partial}{\partial t} .
$$


Such $J$ is called an $\alpha$-admissible almost complex structure on $\operatorname{Symp}(M, \alpha)$. Observe that the Reeb vector field $R_{\alpha}$ satisfies $\omega\left(R_{\alpha}, \cdot\right)=-d\left(e^{t}\right)$, hence is the Hamiltonian vector field of of the function $H: \mathbb{R} \times M \rightarrow \mathbb{R}, H(t, p)=e^{t}$.

Fix a contact quadruple $(M, \xi, \alpha, J)$ so that $J$ is $\alpha$-admissible. We assume that $\alpha$ is regular. Fix a spanning disk $D_{\gamma} \subset M$ of $\gamma$ for each $\gamma \in \mathcal{P}=\mathcal{P}(\alpha)$. Given two Reeb orbits $\gamma_{-}, \gamma_{+}$we denote by $\mathcal{M}_{J}\left(M ; \gamma_{-}, \gamma_{+}\right)$the moduli space of maps $(\tilde{u}, j)$ where

(1) $j$ is an almost complex structure on $\dot{S}^{2}:=S^{2} \backslash\{0, \infty\}$ (here we identify $S^{2}$ with $\left.\mathbb{C} \cup\{\infty\}\right)$;

(2) $\tilde{u}=(a, u):\left(\dot{S}^{2}, j\right) \rightarrow(\mathbb{R} \times M, J)$ is a proper map and is $(j, J)$-holomorphic, ie, $\tilde{u}$ satisfies $d \tilde{u} \circ j=J \circ d \tilde{u}$;

(3) $\tilde{u}$ is asymptotically cylindrical over $\gamma_{-}$at the negative end of $\mathbb{R} \times M$ at the puncture $0 \in S^{2}$; and $\tilde{u}$ is asymptotically cylindrical over $\gamma_{+}$at the positive end of $\mathbb{R} \times M$ at the puncture $\infty \in S^{2}$;

(4) $(\tilde{u}, j) \sim\left(\tilde{v}, j^{\prime}\right)$ if there is a diffeomorphism $f: \dot{S}^{2} \rightarrow \dot{S}^{2}$ such that $\tilde{v} \circ f=$ $\tilde{u}, f_{*} j=j^{\prime}$, and $f$ fixes all punctures.

For generic choice of $J, \mathcal{M}\left(\gamma_{-}, \gamma_{+}\right)=\mathcal{M}_{J}\left(M ; \gamma_{-}, \gamma_{+}\right)$, if not empty, is a smooth manifold,

$$
\operatorname{dim} \mathcal{M}\left(\gamma_{-}, \gamma_{+}\right):=\bar{\gamma}_{+}-\bar{\gamma}_{-}
$$

(recall that $c_{1}(\xi)=0$ ). Such a $J$ is called regular. Note that since $J$ is $\mathbb{R}$-invariant, the $\mathbb{R}$-translation along the $\mathbb{R}$-component of $\mathbb{R} \times M$ induces a free $\mathbb{R}$-action on $\mathcal{M}\left(\gamma_{-}, \gamma_{+}\right)$. If $\tilde{u}=(a, u) \in \mathcal{M}\left(M ; \gamma_{-}, \gamma_{+}\right)$then $u^{*} d \alpha \geq 0$ pointwise. We have

$$
0 \leq E(\tilde{u}):=\int_{\dot{S}^{2}} u^{*} d \alpha=\mathcal{A}_{\alpha}\left(\gamma_{+}\right)-\mathcal{A}_{\alpha}\left(\gamma_{-}\right) .
$$

$E(\tilde{u})$ is called the $d \alpha$-energy of $\tilde{u} . E(\tilde{u})=0$ iff $\gamma_{-}=\gamma_{+}$, and in this case the moduli space consists of a single element $\mathbb{R} \times \gamma_{+}$.

We now proceed to define the cylindrical contact homology of a contact manifold $(M, \xi)$. For a regular contact 1 -form $\alpha$ defining $\xi$ we define the associated cylindrical contact complex $C(\alpha)=\underset{k \in \mathbb{Z}}{\oplus} C_{k}(\alpha)$ to be the graded vector space over $\mathbb{Q}$ generated by elements of $\mathcal{P}=\mathcal{P}(\alpha)$, where $C_{k}(\alpha)$ is the vector space spanned by elements $\gamma \in \mathcal{P}$ with $\bar{\gamma}=k$.

Now we fix a regular $\alpha$-admissible almost complex structure and define the boundary map $\partial: C_{*}(\alpha) \rightarrow C_{*-1}(\alpha)$ as follows. Let $m(\gamma)$ denote the multiplicity of $\gamma \in \mathcal{P}$, then

$$
\partial \gamma:=m(\gamma) \sum_{\sigma \in \mathcal{P}, \bar{\sigma}=\bar{\gamma}-1} n_{\gamma, \sigma} \sigma
$$


where $n_{\gamma, \sigma}$ is the algebraic number of elements of $\mathcal{M}(\sigma, \gamma) / \mathbb{R}$, each element $C \in \mathcal{M}(\sigma, \gamma) / \mathbb{R}$ is weighted by $\frac{1}{m(C)}$, where $m(C)$ is the multiplicity of $C$. Then extend $\partial \mathbb{Q}$-linearly over $C(\alpha)$. Note that since $\alpha$ is regular, $\sigma$ and $\gamma$ are non-degenerate, $\mathcal{M}(\sigma, \gamma) / \mathbb{R}$ is compact and hence a finite set. Moreover, for any $\gamma \in \mathcal{P}$ there are only finitely many $\sigma$ with $\mathcal{A}_{\alpha}(\sigma)<\mathcal{A}_{\alpha}(\gamma)$. Thus $\partial \gamma$ is a finite sum.

We have the following theorem (see [33] and Remark 1.9.2 of [12]).

Theorem 2.1 Let $(\alpha, J)$ be a regular pair. Then $\partial \circ \partial=0$ if $C_{1}(\alpha)=0$.

To prove $\partial \circ \partial=0$ one wants to show that if a 2 -dimensional moduli space $\mathcal{M}\left(\gamma_{-}, \gamma_{+}\right)$has nonempty boundary, then its boundary consists of "broken cylinders" $C_{1} \# C_{2}$, where $C_{1} \in \mathcal{M}\left(\gamma, \gamma_{+}\right) / \mathbb{R}, C_{2} \in \mathcal{M}\left(\gamma_{-}, \gamma\right) / \mathbb{R}$ for some $\gamma \in \mathcal{P}$ with $\bar{\gamma}=\overline{\gamma_{+}}-1$. If this is not true then the boundary of $\mathcal{M}\left(\gamma_{-}, \gamma_{+}\right)$will involve holomorphic curves with more than one negative ends. Such curves are elements of some 1-dimensional moduli space $\mathcal{M}\left(\gamma_{-}, \gamma_{1}, \cdots, \gamma_{j} ; \gamma_{+}\right)$with $j \geq 1$, and $\gamma_{-}$, $\gamma_{1}, \ldots, \gamma_{j}$ are Reeb orbits that form the negative ends of the holomorphic curves. But

$$
\operatorname{dim} \mathcal{M}\left(\gamma_{-}, \gamma_{1}, \cdots, \gamma_{j} ; \gamma_{+}\right)=\bar{\gamma}_{+}-\bar{\gamma}_{-}-\sum_{\nu=1}^{j} \bar{\gamma}_{\nu}=2-\sum_{\nu=1}^{j} \bar{\gamma}_{\nu}
$$

which is less than 1 if $C_{1}(\alpha)=0$. So if $C_{1}(\alpha)=0$ then $\partial \circ \partial=0$. We will see later that every SSFC manifold with $\operatorname{dim}>3$ and $c_{1}(\xi)=0$ will have $C_{*}(\alpha)=0$ for all $* \leq 1$.

When $\partial \circ \partial=0$ we define the The $j$-th cylindrical contact homology group of the pair $(\alpha, J)$ to be

$$
H C_{j}(\alpha, J):=\operatorname{ker}\left(\left.\partial\right|_{C_{j}(\alpha)}\right) / \partial\left(C_{j+1}(\alpha)\right) .
$$

The following theorem, analogous to its counterpart in Floer theory, asserts that $H C(\alpha, J)$ is independent of regular pairs $(\alpha, J)$ satisfying $C_{*}(\alpha)=0$ for $*=-1,0,1$, hence is an invariant of of $(M, \xi)$ (see 33]).

Theorem 2.2 Let $\left(\alpha_{0}=f_{0} \alpha, J_{0}\right),\left(\alpha_{1}=f_{1} \alpha, J_{1}\right)$ be two regular pairs. Assume $C_{i}\left(\alpha_{0}\right)=C_{i}\left(\alpha_{1}\right)=0$ for $i=-1,0,1$. Then there exists a natural isomorphism

$$
\phi^{10}: H C_{i}\left(\alpha_{0}, J_{0}\right) \rightarrow H C_{i}\left(\alpha_{1}, J_{1}\right) .
$$

If $\left(\alpha_{2}, J_{2}\right)$ is a third regular pair then

$$
\phi^{20}=\phi^{21} \circ \phi^{10}, \quad \phi^{00}=i d .
$$


The proof of Theorem 2.2 is similar to the proof of the corresponding theorem in Floer theory. Here the required chain homotopies are guaranteed by the existence of smooth functions $f$ on $\mathbb{R} \times M$ such that $d\left(e^{t} f \alpha\right)$ is symplectic on $\mathbb{R} \times M$ and $e^{t} f \alpha$ interpolates $e^{t} \alpha_{0}$ and $e^{t} \alpha_{1}$. Moreover, in a similar fashion one can show that $H C\left(M, \xi_{0}\right) \cong H C\left(M, \xi_{1}\right)$ for isotopic contact structures $\xi_{0}$ and $\xi_{1}$ on $M$.

We remark here that though the condition $C_{*}(\alpha)=0$ for $*=1,0,-1$ looks artificial, it (or similar conditions on $\bar{\mu}$ ) may impose restrictions on the topology of $M$ and even the type of $\xi$. For example, when $\operatorname{dim} M=3$ and $\left.c_{1}(\xi)\right|_{\pi_{2}(M)}=$ 0 it is proved in [19] that if for some $\alpha \bar{\gamma} \geq 2$ for all contractible Reeb orbits of $\alpha$, then $\pi_{2}(M)=0$ and $\xi$ is tight, ie, there exists no embedded disc $D$ in $M$ such that (i) $\partial D$ is tangent to $\xi$, and (ii) $D$ is transversal to $\xi$ along $\partial D$ (see for example [7]).

\section{Contact handles}

In this section we describe some basic models of contact handles. These basic models have been provided and discussed in detail in 34. Since contact handles are building blocks of SFC manifolds we present a similar discussion here but with a focus on the dynamics of Reeb orbits.

The complex $n$-dimensional space $\mathbb{C}^{n}$ together with its standard complex structure $i$ is a Stein manifold. Let $(x, y, z)$ be the standard coordinates of $\mathbb{C}^{n}$ with respect to the decomposition $\mathbb{C}^{n}=\mathbb{R}^{k} \times \mathbb{R}^{k} \times \mathbb{C}^{n-k},(k \leq n) . x=\left(x_{1}, \ldots, x_{k}\right)$, $y=\left(y_{1}, \ldots, y_{k}\right), z=\left(z_{k+1}, \ldots, z_{n}\right), z_{l}=x_{l}+i y_{l}$.

Fix $0 \leq k \leq n$ and define

$$
f_{\mathrm{st}}(x, y, z)=|x|^{2}-\frac{1}{2}|y|^{2}+\frac{1}{4}|z|^{2} .
$$

$f_{\text {st }}$ is a strictly $i$-convex function on $\mathbb{C}^{n}$. Note that the origin 0 is the only critical point of $f_{\text {st }}$, and its Morse index is $k$.

Define

$$
Y_{\text {st }}:=\left(2 x,-y, \frac{z}{2}\right)=\sum_{j=1}^{k}\left(2 x_{j} \frac{\partial}{\partial x_{j}}-y_{j} \frac{\partial}{\partial y_{j}}\right)+\sum_{l=k+1}^{n} \frac{1}{2}\left(x_{l} \frac{\partial}{\partial x_{l}}+y_{l} \frac{\partial}{\partial y_{l}}\right),
$$

$Y_{\text {st }}=\nabla f_{\text {st }}$, the gradient vector field of $f_{\text {st }}$ with respect to the Euclidean metric. Denote by $\omega_{\text {st }}$ the standard symplectic structure $\sum_{j=1}^{n} d x_{j} \wedge d y_{j}$ on $\mathbb{C}^{n}$. We have $L_{Y_{\mathrm{st}}} \omega_{\mathrm{st}}=\omega_{\mathrm{st}} . Y_{\mathrm{st}}$ is a complete Liouville vector field on the symplectic manifold $\left(\mathbb{C}^{n}, \omega_{\mathrm{st}}\right)$. 
Define $\alpha_{\text {st }}:=\omega_{\text {st }}\left(Y_{\text {st }}, \cdot\right) . \alpha_{\text {st }}$ restricts to a contact 1 -form on $\mathcal{H}$ for any hypersurface $\mathcal{H} \subset \mathbb{C}$ transversal to $Y_{\mathrm{st}}$. Note that $\alpha_{\mathrm{st}}=-d f_{\mathrm{st}} \circ i$.

Consider a function $\mathrm{f}: \mathbb{C}^{n} \rightarrow \mathbb{R}$

$$
\mathrm{f}(x, y, z)=b|x|^{2}-b^{\prime}|y|^{2}+\sum_{k+1}^{n} \frac{\left|z_{l}\right|^{2}}{c_{l}^{2}} ; \quad b>b^{\prime}, c_{l}: \text { positive constants. }
$$

0 is the only critical point of $f$

Define $H_{c}:=\{f=c\}$. Then $H_{c} \pitchfork Y_{\text {st }}$ when $c \neq 0$. Denote the punctured level set $H_{c=0}-\{0\}$ by $H_{o}^{\times}$. We have $H_{o}^{\times} \pitchfork Y_{\text {st }}$. So $\alpha_{\text {st }}$ restricts to a contact 1 -form on each of the level sets of $f$, except at the point of origin.

For $c>0 H_{c}$ contains two special submanifolds:

- a $(2 n-k-1)$-dimensional coisotropic ellipsoid $S_{c}^{+}:=\{|y|=0\} \cap H_{c}$; and

- a $(2 n-2 k-1)$-dimensional contact ellipsoid $S_{c}^{*}:=\{|y|=|x|=0\} \cap H_{c}$.

When $c<0$ there is a $(k-1)$-dimensional isotropic sphere $S_{c}^{-}:=\{|x|=0=$ $|z|\} \cap H_{c}$ on $H_{c}$. The normal bundle of $S_{c}^{-}$has the decomposition

$$
\mathcal{N}\left(S_{c}^{-}, H_{c}\right)=\mathcal{C} \mathcal{S N}\left(S_{c}^{-}, H_{c}\right) \oplus T^{*} S_{c}^{-} \oplus \mathbb{R} R_{\mathrm{f}}
$$

where $\mathcal{C S N}\left(S_{c}^{-}, H_{c}\right)$ is the conformal symplectic normal bundle (see 34]) of $S_{c}^{-} \subset H_{c}, R_{\mathrm{f}}$ is the Reeb vector field of $\left(H_{c}, \alpha_{\mathrm{st}}\right)$.

Note that the vector bundle $T^{*} S_{c}^{-} \oplus \mathbb{R} R_{\mathrm{f}} \cong S_{c}^{-} \times \mathbb{R}^{k}$ is trivial and has a natural framing $\left\{\frac{\partial}{\partial x_{1}}, \ldots, \frac{\partial}{\partial x_{k}}\right\}$. The vector bundle $\mathcal{C} \mathcal{S N}\left(S_{c}^{-}, H_{c}\right)$ is also trivial (of rank $2(n-k))$, and has a natural framing $\mathcal{F}_{k}=\left\{\frac{\partial}{\partial x_{k+1}}, \frac{\partial}{\partial y_{k+1}}, \ldots, \frac{\partial}{\partial x_{n}}, \frac{\partial}{\partial y_{n}}\right\}$.

When $c \rightarrow 0, S_{c}^{+}$(or $S_{c}^{-}$) degenerates to the point 0 .

We denote by $\xi_{c}$ (resp. $\left.\xi_{o}\right)$ the corresponding contact structure on $\left(H_{c}, \alpha_{\mathrm{st}}\right)$ (resp. $\left.\left(H_{o}^{\times}, \alpha_{\mathrm{st}}\right)\right)$.

Proposition 3.1 Let $\mathrm{f}$ and $\overline{\mathrm{f}}$ be two quadratic (up to an addition of a constant) functions of index $k$ with respective coefficients $\left(b, b^{\prime}, c_{j}\right)$ and $\left(\bar{b}, \bar{b}^{\prime}, \bar{c}_{j}\right)$ satisfying conditions in (4). Then (12) implies that for any level sets $H_{c}$ of $\mathrm{f}$ and any level set $\bar{H}_{\bar{c}}$ of $\overline{\mathrm{f}}$ the flow of $Y_{\text {st }}$ will induce

(1) a contact isotopy between $\left(H_{c}, S_{c}^{+}, S_{c}^{*}, \xi_{c}\right)$ and $\left(\bar{H}_{\bar{c}}, \bar{S}_{\bar{c}}^{+}, \bar{S}_{\bar{c}}^{*}, \bar{\xi}_{\bar{c}}\right)$ when $c>$ 0 and $\bar{c}>0$;

(2) a contact isotopy between $\left(H_{c}, S_{c}^{-}, \xi_{c}\right)$ and $\left(\bar{H}_{\bar{c}}, \bar{S}_{\bar{c}}^{-}, \bar{\xi}_{\bar{c}}\right)$ when $c<0$ and $\bar{c}<0$ 
(3) a contact isotopy between $\left(H_{c}-S_{c}^{+}, \xi_{c}\right)$ and $\left(\bar{H}_{\bar{c}}-\bar{S}_{\bar{c}}^{-}, \bar{\xi}_{\bar{c}}\right)$ when $c>0$ and $\bar{c}<0$.

In particular up to contact isotopy, the contact structures on $\left(H_{c}^{ \pm}, \alpha_{\mathrm{st}}\right)$ do not depend on the choice of the coefficients $b, b^{\prime}$, and $c_{j}$; the flow of $Y_{\text {st }}$ will produce the required contact isotopies that even preserve submanifolds like $S_{c}^{ \pm}$and $S_{c}^{*}$. We hence have the freedom to adjust the values of $b, b^{\prime}$ and $c_{j}$ to get Reeb vector fields with desired dynamical behavior.

For notational simplicity we will from time to time use the following symbols: $\left(H_{+}, S_{+}, S_{*}, \xi_{+}\right)$to represent $\left(H_{c}, S_{c}^{+}, S_{c}^{*}, \xi_{c}\right)$ when $c>0$; and $\left(H_{-}, S_{-}, \xi_{-}\right)$to represent $\left(H_{c}, S_{c}^{-}, \xi_{c}\right)$ when $c<0 .\left(H_{+}, \xi_{+}\right)$is called (a standard model of) a contact $k$-handle. It is subcritical if $k<n$.

We now study the Hamiltonian and Reeb dynamics on level sets $H_{ \pm}, H_{o}$, of $\left(\mathbb{C}^{n}, \omega_{\mathrm{st}}, Y_{\mathrm{st}}, \mathrm{f}\right)$. Again $\alpha_{s t}$ is used as the preferred contact 1-form. Let $X_{\mathrm{f}}$ denote the Hamiltonian vector field of $f$ with respect to $\omega_{\text {st }}$,

$$
X_{\mathrm{f}}=\sum_{j=1}^{k}\left(2 b x_{j} \frac{\partial}{\partial y_{j}}+2 b^{\prime} y_{j} \frac{\partial}{\partial x_{j}}\right)+\sum_{l=k+1}^{n} \frac{2}{c_{l}^{2}}\left(x_{l} \frac{\partial}{\partial y_{l}}-y_{l} \frac{\partial}{\partial x_{l}}\right) .
$$

The Reeb vector fields on $\left(H_{o}^{\times}, \alpha_{\mathrm{st}}\right)$ and $\left(H_{ \pm}, \alpha_{\mathrm{st}}\right)$ are

$$
R_{\mathrm{f}}:=\frac{X_{\mathrm{f}}}{\alpha_{\mathrm{st}}\left(X_{\mathrm{f}}\right)}
$$

where

$$
\alpha_{\text {st }}\left(X_{\mathrm{f}}\right)=4 b|x|^{2}+2 b^{\prime}|y|^{2}+\sum_{k+1}^{n}\left|z_{l}\right|^{2} / c_{l}^{2}=3 b|x|^{2}+3 b^{\prime}|y|^{2}+c
$$

is positive away from the point of origin. $R_{\mathrm{f}}$ and $X_{\mathrm{f}}$ have the same integral trajectories up to a reparametrization. Let $\gamma:\left[0, T_{h}\right] \rightarrow \mathbb{C}^{n}$ be a periodic $X_{\mathrm{f}}$ trajectory such that $\dot{\gamma}(t)=X_{\mathrm{f}}(\gamma(t))$. $T_{h}$ is then called the Hamiltonian period of $\gamma$. The Reeb period of $\gamma$ can be defined similarly, and is actually its action $\int_{\gamma} \alpha_{\mathrm{st}}$.

Lemma 3.1 (i) There is no periodic Reeb trajectory on $H_{o}^{\times}$and $H_{-}$.

(ii) On $H_{+}$all periodic Reeb trajectories are contained in $S_{*}$.

(iii) If $c_{k+1}^{2}, \ldots, c_{n}^{2}$ are linearly independent over $\mathbb{Q}$ then the Hamiltonian period of a simple periodic trajectory on $H_{c}$ with $c>0$ is $\pi c_{l}^{2}$ for some $k<l \leq n$, while its action is $\pi c_{l}^{2} c$. 
Proof Let $\varphi_{t}(w)=\varphi(t, w): \mathbb{R} \times \mathbb{C}^{n} \rightarrow \mathbb{C}^{n}, \varphi(0, w)=i d$ be the flow of $X_{\mathrm{f}}$, $\gamma(t)=(x(t), y(t), z(t))$ an integral trajectory of $X_{\mathfrak{f}}$ with $\dot{\gamma}(t)=X_{\mathbf{f}}(\gamma(t))$. Then on $\gamma$

$$
\begin{aligned}
& \dot{x}_{j}=2 b^{\prime} y_{j}, \quad \dot{y}_{j}=2 b x_{j}, \quad \text { for } \quad 1 \leq j \leq k \\
& \dot{x}_{l}=2 y_{l} / c_{l}^{2}, \quad \dot{y}_{l}=-2 x_{l} / c_{l}^{2}, \quad \text { for } \quad k+1 \leq l \leq n
\end{aligned}
$$

We have

$$
\left\{\begin{array}{l}
x_{j}(t)=x_{j}(0) \cosh \left(2 \sqrt{b b^{\prime}} t\right)+y_{j}(0) \sqrt{\frac{b^{\prime}}{b}} \sinh \left(2 \sqrt{b b^{\prime}} t\right) \\
y_{j}(t)=y_{j}(0) \cosh \left(2 \sqrt{b b^{\prime}} t\right)+x_{j}(0) \sqrt{\frac{b}{b^{\prime}}} \sinh \left(2 \sqrt{b b^{\prime}} t\right) \\
x_{l}(t)=x_{l}(0) \cos \left(2 t / c_{l}^{2}\right)-y_{l}(0) \sin \left(2 t / c_{l}^{2}\right) \\
y_{l}(t)=y_{l}(0) \cos \left(2 t / c_{l}^{2}\right)+x_{l}(0) \sin \left(2 t / c_{l}^{2}\right)
\end{array}\right.
$$

for $1 \leq j \leq k$ and $k+1 \leq l \leq n$. $\left(x_{j}(t), y_{j}(t)\right)$, if not identically zero, is hyperbolic, while $\left|z_{l}(t)\right|$ is a constant along any $\gamma$. So $\gamma$ is contained in $S_{*}$ if it is periodic. Hence (i) and (ii) are true.

Assume $\gamma \subset S_{*}$. The Hamiltonian period of the $z_{l}$-component of $\gamma$ is $\pi c_{l}^{2}$. Hence if $c_{k+1}^{2}, \ldots, c_{n}^{2}$ are linearly independent over $\mathbb{Q}$ then for any $c>0$ there are only $n-k$ simple periodic trajectories on $H_{c}$. They are $\sigma_{l}:=\left\{\left|z_{l}\right|^{2}=c_{l}^{2} c\right\}$, $l=k+1, \ldots, n$. The Hamiltonian period of $\sigma_{l}$ is $\pi c_{l}^{2}$, which is independent of the value of $c$, while the action of $\sigma_{l}$ is $\pi c_{l}^{2} c$. This proves (iii).

Note that actions of simple Reeb orbits can be made as small as we want by choosing $c_{l}^{2}$ to be small enough.

Theorem 3.1 Let $H_{+}$be as above.

(I) All periodic Reeb orbits of $H_{+}$are "good" as defined in (3).

(II) If $c_{k+1}^{2}, \ldots, c_{n}^{2}$ are linearly independent over $\mathbb{Q}$ then all Reeb orbits of $H_{c}$ are non-degenerate.

$$
C_{*}\left(H_{+}, \alpha_{\mathrm{st}}\right)=0 \text { if } *<2 n-k-2 \text { or } *-(2 n-k-2) \text { is odd. }
$$

(IV) Can choose (or deform) $H_{+}$for any given $m_{o}>0$ such that for $* \leq m_{o}$

$$
r k\left(C_{*}\left(H_{+}, \alpha_{\mathrm{st}}\right)\right)= \begin{cases}1 & \text { if } *=2 n-k-4+2 i \text { for some } i \in \mathbb{N} \\ 0 & \text { otherwise. }\end{cases}
$$

We start with the following lemma: 
Lemma 3.2 Let $\gamma$ be a contractible Reeb orbit of a contact manifold $(M, \xi)$ with contact 1 -form $\alpha$. Let $D \subset M$ be a spanning disc of $\gamma$. Then

$$
\mu\left(\gamma,\left.\xi\right|_{D}\right)=\mu\left(\gamma,\left.\left(\mathbb{C} R_{\alpha} \oplus \xi\right)\right|_{D}\right)
$$

where $R_{\alpha}$ is the Reeb vector field of $\alpha$.

Proof of Lemma 3.2 Let $\varphi:\left.\mathbb{C} R_{\alpha}\right|_{D} \stackrel{\sim}{\rightarrow} \mathbb{C} \times D$ be the vector bundle isomorphism $\varphi\left(\lambda R_{\alpha}, p\right)=(\lambda, p)$ for $\lambda \in \mathbb{C}$ and $p \in D . \varphi$ is a symplectic trivialization of the vector bundle $\left.\mathbb{C} R_{\alpha}\right|_{D}$. The action of the linearized Reeb flow on $\left.\mathbb{C} R_{\alpha}\right|_{\gamma}$ is a constant path (ie, a point) in $S p(2)$ with respect to $\varphi$. Let $\Phi$ be any symplectic trivialization of $\xi$ over $D$, then $\varphi \oplus \Phi$ is a symplectic trivialization of $\mathbb{C} R_{\alpha} \oplus \xi$ over $D$. The definition of $\varphi$ implies that $\mu\left(\gamma,\left.\xi\right|_{D} ; \Phi\right)=\mu\left(\gamma,\left.\left(\mathbb{C} R_{\alpha} \oplus \xi\right)\right|_{D} ; \varphi \oplus \Phi\right)$. Since the $\mu$-index is independent of the choice of a symplectic trivialization over a fixed spanning disc, we conclude that $\mu\left(\gamma,\left.\xi\right|_{D}\right)=\mu\left(\gamma,\left.\left(\mathbb{C} R_{\alpha} \oplus \xi\right)\right|_{D}\right)$.

Proof of Theorem 3.1 Let $\Phi:=T \mathbb{C}^{n} \sim \mathbb{C}^{n} \times \mathbb{C}^{n}$ be the standard trivialization of the tangent bundle $T \mathbb{C}^{n}$ of $\mathbb{C}^{n}$. When restricted on $H_{+}, \Phi$ is a trivialization of the stabilized contact bundle $\mathbb{C} R \oplus \xi=T_{H_{+}} \mathbb{C}^{n}$. Here $R=R_{\mathrm{f}}$ is the Reeb vector field of $\alpha_{\text {st }}$ on $H_{+}$.

By Lemma 3.2 we can use $\Phi$ to compute the $\mu$-index of any $R_{\mathrm{f}}$-orbit $\sigma$ in $H_{+}$. Moreover, $\pi_{2}\left(H_{+}\right)=0$ if $\operatorname{dim} H_{+}>3$. When $\operatorname{dim} H_{+}=3, \pi_{2}\left(H_{+}\right)$is generated by $S_{+}$. The inclusion $H_{+} \hookrightarrow \mathbb{C}^{2}$ implies that $\left.\left(\xi_{+} \oplus \mathbb{C} R\right)\right|_{S_{+}}$and $T_{S_{+}} \mathbb{C} \cong \mathbb{C}^{2} \times S_{+}$are isomorphic vector bundles over $S_{+}$. Since $\left.\mathbb{C} R\right|_{S_{+}}$is a trivial bundle over $S_{+}$, so is $\left.\xi_{+}\right|_{S_{+}}$, which implies that $c_{1}\left(\xi_{+}\right)=0$. Therefore the index $\mu\left(\sigma, D_{\sigma}\right)$ is independent of the choice of the spanning disk $D_{\sigma}$ in $H_{+}$.

Extend the linearized Reeb flow $R_{*}^{t}$ of $R$ to $T_{H_{+}} \mathbb{C}^{n}$ by assigning $R_{*}^{t}\left(Y_{\mathrm{st}}\right)=$ $Y_{\text {st }} \circ R^{t}$. We have (with respect to $\Phi$ )

$$
\left.R_{*}^{t}\right|_{\sigma_{l}}=e^{t D}=\left(\begin{array}{ccc}
e^{t D_{1}} & & \\
& \ddots & \\
& & e^{t D_{n}}
\end{array}\right) \in S p(2 n)
$$

where

$$
D_{j}=\left(\begin{array}{cc}
0 & 2 b^{\prime} \\
2 b & 0
\end{array}\right) \quad, \quad D_{\ell}=\frac{2}{c c_{\ell}^{2}}\left(\begin{array}{cc}
0 & -1 \\
1 & 0
\end{array}\right) \in G l(2, \mathbb{R})
$$

for $j=1, \ldots k, \ell=k+1, \ldots n$.

By easy computation one finds that for $j=1, \ldots, k$, 


$$
e^{t D_{j}}=\left(\begin{array}{cc}
\cosh 2 \sqrt{2 b b^{\prime}} t & \sqrt{\frac{b^{\prime}}{b} \sinh 2 \sqrt{b b^{\prime}} t} \\
\sqrt{\frac{b}{b^{\prime}}} \sinh 2 \sqrt{2 b b^{\prime}} t & \cosh 2 \sqrt{b b^{\prime}} t
\end{array}\right)
$$

with $\operatorname{det}\left(I-e^{t D_{j}}\right)<0$ for all $t \neq 0$. Moreover, each $e^{t D_{j}}$ has two real positive eigenvalues $\cosh 2 \sqrt{b b^{\prime}} t \pm \sinh 2 \sqrt{b b^{\prime}} t$ for all $t$. Therefore $e^{t D_{j}}$ makes no rotations to the $\left(x_{j}, y_{j}\right)$-plane, hence has no contribution to $\mu\left(\sigma_{l}\right)$.

For $\ell=k+1, \ldots, n$, we have

$$
e^{t D_{\ell}}=\left(\begin{array}{cc}
\cos \theta & -\sin \theta \\
\sin \theta & \cos \theta
\end{array}\right), \text { where } \theta=\frac{2 t}{c c_{\ell}^{2}} .
$$

Note that $\operatorname{det}\left(I-e^{t D_{\ell}}\right) \geq 0$, the equality holds if and only if $t$ is an integral multiple of $\pi c c_{\ell}^{2}$.

We have for a simple Reeb orbit $\sigma$ in $H_{+}$:

(1) $\bar{\sigma} \geq(n-k-1)+2+(n-3)=2 n-k-2$. The minimum is always achieved by some $\sigma$.

(2) For $m, m^{\prime} \in \mathbb{N}$ with $m>m^{\prime}, \overline{\sigma^{m}}-\overline{\sigma^{m^{\prime}}}$ is a positive even number, and is $\geq 2\left(m-m^{\prime}\right)$. Here $\sigma^{m}$ denotes the $m$-th multiple of $\sigma$.

This proves Part (I) and (III) of the theorem.

If we choose to have $c_{k+1}^{2}, c_{k+2}^{2}, \ldots, c_{n}^{2}$ linearly independent over $\mathbb{Q}$, then there are exactly $n-k$ simple Reeb orbits $\sigma_{k+1}, \ldots, \sigma_{n}$ as defined before. From the computations above it is easy to see that these $\sigma_{l}$ and their multiple covers are all non-degenerate. So Part (II) is true.

To prove Part (IV) we consider the following perturbation of $c_{l}$ to compute indexes. For any (large) integer $n_{o} \in \mathbb{N}$, choose $c_{k+1}, \ldots, c_{n}$ such that $c_{k+1}^{2}$, $\ldots, c_{n}^{2}$ are linearly independent over $\mathbb{Q}$, and $n_{o} c_{n}^{2}<c_{l}^{2}$ for $l=k+1, \ldots, n-1$. Then

$$
\begin{aligned}
& \overline{\sigma_{n}^{m}}=2 n-k-4+2 m \text { for } 1 \leq m \leq n_{o}, \\
& \overline{\sigma_{n}^{m}} \geq 2 n-k-4+2 m \text { for } m>n_{o}, \\
& \overline{\sigma_{l}^{m}} \geq 2 n-k-4+2\left(n_{o}+m\right) \text { for } l>k+1, m \geq 1 .
\end{aligned}
$$

Now choose $n_{o}$ so that $n_{o}>m_{o}$. This completes the proof of Part (IV).

Remark 3.1 We call the Reeb orbit $\sigma_{n}$ corresponding to $c_{n}$ the principal (Reeb) periodic trajectory or the principal (Reeb) orbit of $\left(H_{+}, \alpha_{\mathrm{st}}\right)$ if (10) is satisfied. When $m_{o} \rightarrow \infty$ the contact complex $C_{*}\left(H_{+}, \alpha_{\mathrm{st}}\right)$ is essentially generated 
by $\sigma_{n}$ and its positive multiples. We call each positive multiple of $\sigma_{n}$ a principal generator of $C_{*}\left(H_{+}, \alpha_{\mathrm{st}}\right)$. We see that $C\left(H_{+}, \alpha_{\mathrm{st}}\right)$ stabilizes as $m_{o} \rightarrow \infty$, where $C_{*}\left(H_{+}, \alpha_{\mathrm{st}}\right)$ is a vector space of rank 1 precisely when $*=2 n-k-4+2 j$ for some $j \in \mathbb{N}, \ldots$, otherwise it is 0 .

We now proceed to study the local index of a non-periodic Reeb trajectory $\gamma$ on $H_{+}$. Recall that our contact $k$-handle is modelled on the following hypersurface in $\mathbb{C}^{n}$ :

$$
b|x|^{2}-b^{\prime}|y|^{2}+\sum_{k+1}^{n} \frac{\left|z_{l}\right|^{2}}{c_{l}^{2}}=c>0
$$

Since later we will see that a SSFC manifold can be constructed by attaching thin subcritical contact handles to a tiny tubular neighborhood of attaching isotropic spheres, we are mainly interested in the domain $U_{+} \subset H_{+}$(a tubular neighborhood of the belt sphere of $H_{+}$) where $b^{\prime}|y|^{2} \leq C$ for some constant $C>0,|x|^{2}+|z|^{2}$ is small.

Recall the Hamiltonian vector field $X_{\mathrm{f}}$ and Reeb vector field $R_{\mathrm{f}}=X_{\mathrm{f}} / \alpha_{\mathrm{st}}\left(X_{\mathrm{f}}\right)$ from (5), (6) and (7). Recall that the standard trivialization of the tangent bundle $T \mathbb{C}^{n}$ induces a symplectic trivialization (with respect to $\omega_{\text {st }}$ ) $\Phi$ of the stabilized bundle $\mathbb{C} R_{\mathrm{f}} \oplus \xi_{+}$of $\xi_{+}$.

View $\gamma$ as a non-periodic $X_{\mathfrak{f}}$-trajectory on $U_{+}$with Hamiltonian period $T_{h}$, and reparametrize $\gamma$ so that $\dot{\gamma}(t)=X_{\mathbf{f}}(\gamma(t))$. Since $H_{+}$is subcritical, given any positive number $N_{o}$ we can have

$$
\mu\left(\gamma(t), \tilde{\xi}, \Phi_{\mathrm{st}}\right)>N_{o} T_{h}
$$

by thinning $U_{+}$, ie, by choosing to have $c_{l}^{2}$ small enough.

Now we parametrize $\gamma$ as an $R_{\mathrm{f}}$-trajectory, ie, $\dot{\gamma}(\tau)=R_{\mathrm{f}}(\gamma(\tau))$. Since on $U_{+}$

$$
\alpha_{\mathrm{st}}\left(X_{\mathrm{f}}\right)=3 b|x|^{2}+3 b^{\prime}|y|^{2}+c \leq 6 C+4 c
$$

the action $T$ of $\gamma$ satisfies

$$
T \leq C_{o} T_{h}, \quad C_{o}=6 C+4 c .
$$

Denote by $\psi$ the flow of $R_{\mathrm{f}}$, and $\varphi$ the flow of $X_{\mathrm{f}}$. We have

$$
\psi(\tau, w)=\varphi(t(\tau, w), w)
$$

Both flows preserve $\xi$ and have the following relation between their linearized flows:

$$
d \psi(\tau, w)=d \varphi(t(\tau, w), w)+\frac{d \varphi}{d t}(t(\tau, w), w) \otimes d t
$$


where $\frac{d \varphi}{d t}(t, w)=X_{\mathrm{f}} \circ \varphi(t, w)$. The term $\frac{d \varphi}{d t} \otimes d t=X_{\mathrm{f}} \otimes d t$ is a path of $2 n \times 2 n$ matrices of rank 1 and has $\mu$-index equal to 0 . Hence by [32] we have

$$
\begin{gathered}
\left|\mu\left(\gamma(\tau), \tilde{\xi}, \Phi_{\mathrm{st}}\right)-\mu\left(\gamma(t), \tilde{\xi}, \Phi_{\mathrm{st}}\right)\right| \leq 2 n, \\
\mu\left(\gamma(\tau), \xi, \Phi_{\mathrm{st}}\right)=\mu\left(\gamma(\tau), \tilde{\xi}, \Phi_{\mathrm{st}}\right) \geq \mu\left(\gamma(t), \tilde{\xi}, \Phi_{\mathrm{st}}\right)-2 n>N_{o} T_{h}-2 n
\end{gathered}
$$

So we obtain a linear inequality relating the action $T$ of a Reeb trajectory $\gamma$ and its $\mu$-index:

$$
\mu\left(\gamma(\tau), \xi, \Phi_{\mathrm{st}}\right)>N T-2 n, \quad N=C_{o}^{-1} N_{o}
$$

$N$ can be made very large by thinning the subcritical handle. Here the fact that $H_{+}$is subcritical is essential to the largeness of $N$. We summarize the above discussion about non-periodic trajectories in the following lemma:

Lemma 3.3 Let $U_{+}$be a tubular neighborhood of the belt sphere of a $(2 n-1)-$ dimensional subcritical contact $k$-handle $H_{+}$. Let $N$ be any positive number. Then by thinning $U_{+}$, ie, by choosing to have $c_{l}^{2}(l=k+1, \ldots, n)$ small enough, we have

$$
\mu\left(\gamma, \xi, \Phi_{\mathrm{st}}\right)>N T-2 n
$$

for any non-periodic Reeb trajectory of $\alpha_{\text {st }}$ on $U_{+}$with action $T$.

\section{Reeb dynamics on SSFC manifolds}

A closed, orientable $(2 n-1)$-dimensional contact manifold $(M, \xi)$ is called Stein-fillable if there is a $2 n$-dimensional Stein domain $(V, J)$ such that $\partial V=$ $M$ and $\xi$ is the maximal complex subbundle of $T M .(V, J)$ is called a Stein filling of $(M, \xi)$. Let $f$ be a strictly $J$-convex function on $V$ that extends smoothly to the boundary $\partial V=M$ as a constant function, then $\omega_{f}:=$ $-d d^{J} f=-d(d f \circ J)$ is a symplectic 2 -form on $\bar{V}$ (the nondegeneracy of $\omega_{f}$ is ensured by the strict $J$-convexity of $f$ ), and $\xi$ is the kernel of the restriction of the 1 -form $\alpha_{f}:=\omega(\nabla f, \cdot)$ on $T M$. Here $\nabla f$ is the gradient vector field of $f$ with respect to the Riemannian metric $g(\cdot, \cdot):=\omega_{f}(\cdot, J \cdot)$, and hence is a Liouville vector field of $\omega_{f}$. Without loss of generality we may assume that $f$ is also a Morse function. We call a Stein-fillable $(M, \xi)$ subcritical if the corresponding $f$ is subcritical, ie, has no critical points of index $\geq n$. Notice that $\left(V, \omega_{f}, \nabla f, f\right)$ is actually an open domain of a Weinstein manifold. In the following we will study the Reeb dynamics of SSFC manifolds in the setup of Weinstein manifolds.

A Weinstein manifold is a quadruple $(W, \omega, Y, f)$ where 
- $(W, \omega)$ is a symplectic manifold,

- $Y$ is a complete smooth vector field on $W$, and $Y$ is a Liouville vector field of $(W, \omega)$, ie,

$$
L_{Y} \omega=\omega
$$

where $L_{Y} \omega$ denotes the Lie derivative of $\omega$ with respect to $Y$,

- $f$ is an exhausting Morse function on $W$, and $Y$ is gradient-like with respect to $f$, ie, $d f(Y)>0$ except at critical points of $f$.

In this paper we are interested in Weinstein manifolds of finite type where the function $f$ has only finitely many critical points, and $Y$ has only finitely many zeros accordingly.

A Weinstein manifold $(W, \omega, Y, f)$ associates a 1 -form $\alpha:=\omega(Y, \cdot)$ which is a primitive of $\omega$. Let $S \subset W$ be a hypersurface transversal to $Y$, then $\alpha$ restricts to a contact 1 -form on $S$. Let $X$ be a nonvanishing vector field which span the line field $\mathcal{L}_{S} \subset T S$ on which $\omega$ degenerates. Then the Reeb vector field of $\left(S,\left.\alpha\right|_{T S}\right)$ is $R:=X / \alpha(X)$. If $S$ is also a level set of a function $h$, then $R=X_{h} / \alpha\left(X_{h}\right)$, where $X_{h}$, satisfying $\omega\left(X_{h}, \cdot\right)=-d h$, is the Hamiltonian vector field associated to $h$.

Let $S^{\prime} \subset W$ be another hypersurface transversal to $Y$. Let $\zeta$ and $\zeta^{\prime}$ be contact structures on $S$ and $S^{\prime}$ defined by $\alpha$ respectively. If a reparametrized flow of $Y$ induces a diffeomorphism $\varphi: S \rightarrow S^{\prime}$ then we have $\varphi_{*} \zeta=\zeta^{\prime}$, hence $(S, \zeta)$ and $\left(S^{\prime}, \zeta^{\prime}\right)$ are contactomorphic. This is because for any two smooth functions $h_{1}, h_{2}>0$ on $W$, we have

$$
L_{h_{1} Y} h_{2} \alpha=h_{1}\left(d h_{2}(Y)+h_{2}\right) \alpha .
$$

Note that we have $\alpha(Y)=0$ by definition.

Now let $f$ be a subcritical strictly $J_{W}$-convex Morse function on a Stein manifold $\left(W, J_{W}\right)$. If $W$ is of finite type, then $\omega:=\omega_{f}$ is independent of the choice of $f$ up to a diffeomorphism of $W$. Let $Y_{f}=\nabla f$. The quadruple $\left(W, \omega_{f}, Y_{f}, f\right)$ is then a Weinstein manifold. It is easy to see that a contact manifold $(M, \xi)$ is subcritical Stein-fillable (up to contact isotopy) iff it can be realized as a hypersurface in some subcritical $\left(W, \omega_{f}, Y_{f}, f\right)$ that is also transversal to $Y_{f}$, or equivalently, a regular level set of $f$. For any level set $Q$ of $f, \alpha_{f}:=\omega\left(Y_{f}, \cdot\right)$ restricted to a contact 1 -form on $Q$ away from critical points of $f$. Moreover the Reeb vector field associated to $\alpha$ and the Hamiltonian vector field of $f$ have the same integral trajectories.

We now proceed to study the Reeb dynamics on level sets of $\left(W, \omega_{f}, Y_{f}, f\right)$. First of all, a theorem of Eliashberg [9] states that one can manipulate critical 
points $f$ as freely as in the smooth case. Thus $f$ can be assumed to has only one critical point of index 0 (we assume that $W$ is connected), and $f(p)<f(q)$ for $p, q \in C r i t(f)$ if the Morse index of $p$ is less than the Morse index of $q ; f(p)=f(q)$ if $p, q \in C r i t(f)$ are of the same index. Also, following 8 a subcritical Stein manifold $W$ of dimension $2 n \geq 4$ can reconstructed by attaching handles of index $<n$. Therefore, once a subcritical $J$-convex Morse function $f$ on $\left(W, J_{W}\right)$ (of finite type) is chosen, $\left(W, J_{W}\right)$ can be decomposed into a (finite) union of handlebodies of subcritical indexes accordingly. A critical point of Morse index $k$ corresponds to exactly a handlebody of of index $k$, and $\left(W, J_{W}\right)$ can be constructed by attaching back these handlebodies along isotropic spheres with specified framings in the order of handle indexes.

A $2 n$-dimensional handlebody of index $k$ is diffeomorphic to $D^{k} \times D^{2 n-k}$ with boundary $S^{k-1} \times D^{2 n-k} \cup D^{k} \times D^{2 n-k-1}$. $S^{k-1} \times D^{2 n-k}$ is to be glued, while $D^{k} \times S^{2 n-k-1}$ is a contact $k$-handle. Thus a SSFC manifold can be constructed by attaching subcritical contact handles modelled on a tubular neighborhood $U_{+}$of the belt sphere of $H_{+}=\left\{b|x|^{2}-b^{\prime}|y|^{2}+\sum_{l=k+1}^{n}\left|z_{l}\right|^{2} / c_{l}^{2}=c\right\}$ (see [5] for more detail). We may assume that contact handles of $(M, \xi)$ of the same index are pairwise disjoint.

Recall that each subcritical contact $k$-handle has only $n-k$ simple Reeb orbits. We may assume that all attaching $(k-1)$-spheres miss all the simple Reeb orbits in the middle of contact handles of lower indexes. Thus a SSFC manifold has two types of contractible Reeb orbits. Type I Reeb orbits are those contained in the middle of subcritical contact handles; Reeb orbits which are not of type I are called Type II. Type II orbits run through different handles.

Lemma 4.1 (See Lemma 3 of [5]) Let $(M, \xi)$ be a SSFC manifold with a contact handle decomposition. Let $T$ be any positive number. Then up to a contact isotopy there is a defining contact 1 -form of $(M, \xi)$ so that any Reeb trajectory which leaves a contact $k$-handle and return to possibly another contact $k$-handle has action $\geq T$.

Here is a brief explanation of why Lemma 4.1 is true. The attaching isotropic spheres of subcritical contact $k$-handles are of dimension less than (dim $M-$ $1) / 2$, hence after isotopy we may assume that there are no Reeb chords connecting these spheres. So for any $T>0$, there is a neighborhood $\mathcal{U}_{k}$ of these spheres such that any Reeb trajectory leaves $\mathcal{U}_{k}$ at time 0 will not meet $\mathcal{U}_{k}$ again before time $T$. Now we glue the contact $k$-handles to the interior of $\mathcal{U}_{k}$.

By combining the proof of Proposition 1 in [5] and an estimate of $\bar{\mu}$-index based on Lemma 4.4 and the analysis on handles in the previous section we 
can derive the following lemma concerning the $\bar{\mu}$-index of contractible Type II Reeb orbits.

Lemma 4.2 Let $(M, \xi)$ be a SSFC manifold with a subcritical contact handle decomposition. Let $K$ be any positive number. Then up to contact isotopy (by thinning handles) every Type II contractible Reeb orbit has $\bar{\mu}$-index greater than $K$.

The rest of this section is devoted to proving Lemma 4.2

We have shown that over each subcritical contact handle there is a linear inequality relating the action $T$ of a Reeb trajectory $\gamma$ and its $\mu$-index. Namely $\mu(\gamma) \geq N \cdot T-2 n, C$ is independent of $\gamma, N$ can be made very large by shaping the subcritical handle. In the following we will estimate the actual $\mu$-index of a contractible Type II orbit, which is related to local indexes, the number of times the orbit crosses different handles, the framings of the symplectic normal bundles of the attaching isotropic spheres, and the gluing process. We will prove that there is a linear relation between the action of a contractible Type II orbit and the number of times it crosses different handles. This linear relation, together with the said linear inequality and the largeness of the action of any Type II orbit, enable us to prove Lemma 4.2

We now make a digression here to prepare for the statement of the inequality that links all local estimates together and guarantees the largeness of $\mu$ - (and hence $\bar{\mu}^{-}$) indexes of Reeb orbits of Type II.

Let $S_{n}:=\{0,1,2, \ldots, n-1\}$ be a set of $n$ "letters", $n \geq 2$. Define $\mathcal{W}_{n}$ to be the set of "words" of finite length whose letters are elements of $S_{n}$.

Definition 4.1 Given $w=l_{1} \cdots l_{m} \in \mathcal{W}_{n}, l_{i} \in S_{n}, w$ is called jumpy if $l_{i} \neq l_{i+1}$ for all $1 \leq i<m$.

Definition 4.2 Given $w \in \mathcal{W}_{n}, w=l_{1} l_{2} l_{3} \cdots l_{m}, w$ contains a basin or has $a$ basin if there is an $k, 0<k<n$ and a subword $w^{\prime} \subset w, w^{\prime}=l_{i} \cdots l_{j}$, $1<i \leq j<m$, such that $l_{i-1}=l_{j+1}=k>l_{\nu}$ for $i \leq \nu \leq j$. $w^{\prime}$ is called a basin of $w$.

Lemma 4.3 (Word Lemma) Any jumpy word $w \in \mathcal{W}_{n}$ of length $2^{n}$ must contain a basin $(n \geq 2)$.

Corollary 4.1 Any jumpy word $w \in \mathcal{W}_{n}$ of length $m$ must contain at least $\left[\frac{m}{2^{n}}\right]$ disjoint basins. 
Proof of the Word Lemma By mathematical induction. When $n=2$, there are only two jumpy words of length 4: 0101 and 1010. Both contain a basin "0" with $k=1$. Hence the lemma is true for $n=2$. Assume the lemma holds for $n=s$. Let $w \in \mathcal{W}_{s+1}$ be jumpy of length $2^{s+1}$. If the largest letter appearing in $w$ is less than s, then it reduces to the case $n=s$ and the statement holds again. If the letter $s$ appears in $W$ at least twice then we are done. If not, then $w=w_{1} s w_{2} . w_{1}, w_{2} \in \mathcal{W}_{s}$ are jumpy. Observe that one of them is of length $\geq 2^{s}$ and hence contains a basin by assumption. This basin is also a basin of $w$. So the lemma is true for $n=s+1$. By induction we conclude that the lemma is true for all $n$ greater than 1 .

Now let $k_{o}$ be the highest index of contact handles of $(M, \xi)$. We may assume that Lemma 4.1 holds true for $(M, \xi)$.

Let $H(k)$ denote th union of all contact $k$-handles of $(M, \xi)$. Define

$$
\mathcal{H}(k):=H(k) \backslash \bigcup_{k^{\prime}>k} H\left(k^{\prime}\right)
$$

Let $\gamma$ be a simple Reeb orbit of Type II. $\gamma$ associates a jumpy word $w(\gamma) \in \mathcal{W}_{n}$ constructed as follows.

The codimension 1 boundaries of $\mathcal{H}(k), k=0, \ldots, k_{o}$, cut $\gamma$ into $m-1$ connected curves with boundaries. Let $\bar{k}>0$ be the maximal value of $k$ such that $\gamma \cap \mathcal{H}(k)$ is not empty. Fix a connected component of $\gamma \cap \mathcal{H}(\bar{k})$ and call it $\gamma_{1}$. Following the Hamiltonian flow starting from $\gamma_{1}$ we write $\gamma$ as the ordered union $\gamma=\cup_{j=1}^{m-1} \gamma_{j}$ of these connected curves $\gamma_{j}$. Define for $j=1, . ., m-1$ that $l_{j}:=k$ if $\gamma_{j} \subset \mathcal{H}(k)$, and $l_{m}:=l_{1}$. Then define $w(\gamma)$ to be $w(\gamma):=l_{1} l_{2} \cdots l_{m}$. $w(\gamma)$ is well-defined up to a choice of $\gamma_{1}$. Clearly $w(\gamma)$ is jumpy and contains at least one basin. By the Word Lemma $w(\gamma)$ contains at least $\left[\frac{m}{2^{n}}\right]$ disjoint basins. Also, following Lemma 4.1 we have the property that, if $l_{i} \cdots l_{j}$ is a basin of $w(\gamma)$ then the action of $\gamma_{i} \cup \cdots \cup \gamma_{j}$ must be greater that $T$ for any prescribed number $T>0$. We then have the following lemma:

Lemma 4.4 For any $T>0$, one can modify a subcritical Stein manifold $(W, \omega, Y, f)$ such that any simple Type II Hamiltonian orbit $\gamma$ with a word $w(\gamma)$ of length $m$ must have action $\mathcal{A}(\gamma)>C_{m} \cdot T$, where $C_{m}=\max \left\{1,\left[\frac{m}{2^{n}}\right]\right\}$.

Recall that each handle is attached along an isotropic $(k-1)$-sphere modelled on $S_{-}$with a specified framing $\mathcal{F}$ of the normal bundle of the sphere. According to Weinstein [34] there is a neighborhood $U$ of $S$ in $W$, a neighborhood $U_{-}$of $S_{-}$in $\mathbb{C}^{n}$, and an isomorphism of isotropic setups

$$
\phi:(U, \omega, Y, M \cap U, S) \rightarrow\left(U_{-}, \omega_{\mathrm{st}}, Y_{\mathrm{st}}, H_{-} \cap U_{-}, S_{-}\right) .
$$


This isomorphism identifies the chosen framing $\mathcal{F}$ of $\mathcal{N}(S, M)$ with the standard framing of $\mathcal{N}\left(S_{-}, H_{-}\right)$. The reparamertized flow of $Y_{\text {st }}$ induces a contactomorphism

$$
\eta: U_{-} \backslash S_{-} \rightarrow U_{+} \backslash S_{+} \subset H_{+},
$$

$H_{+}$is a contact $k$-handle as described in Proposition 3.1 Via the contactomorphism $\eta$ the framing $\mathcal{F}$ induces a symplectic trivialization $\Phi_{\mathcal{F}}$ of $\xi$ over $H_{+} \backslash S_{+}$. Recall also that $\xi$ on $H_{+}$has a symplectic trivialization $\Phi_{\text {st }}$ induced by the standard symplectic trivialization of $T \mathbb{C}^{n}$. We can choose $c_{l}$ so that for any Hamiltonian trajectory $\gamma$ in some $\mathcal{H}(p)$ with $\mathcal{A}(\gamma)=\tau$,

$$
\mu\left(\gamma, \tilde{\xi}_{k}, \Phi_{\mathcal{F}}\right)>\left(N_{1}(\mathbf{c})+N_{2}(\mathbf{c})\right) \tau-2 n
$$

where $N(\mathbf{c})$ is a constant depending only on $c_{l}$ 's and is big enough so that $N(\mathbf{c}) \tau$ exceeds any of the Conley-Zehnder indexes rising from the ambiguities caused by the different symplectic trivializations $\Phi_{\mathcal{F}}$ and $\Phi_{\mathrm{st}} \cdot N_{2}(\mathbf{c})$ is also a very large constant.

Now let $\gamma$ be a simple contractible Type II orbit of $M$. Let $D \subset M$ be a closed spanning disc of $\gamma$. By perturbing the interior of $D$ we may assume the following condition on $D$.

Condition 4.1 Each connected component of the intersection $D \cap \mathcal{H}(k)$ contains a part of $\gamma$ if it is not empty.

Let $w(\gamma)=l_{1} \cdots l_{m}$ be the word associated to $\gamma$. Write $D=\cup_{j=1}^{m-1} D_{j}$, where $D_{j}$ is the intersection of $D$ with the $j$-th handle that $\gamma$ crosses. On each $D_{j}$ we use the symplectic trivialization $\Phi_{j}=\Phi_{\text {st }}$ on $\tilde{\xi}$ and compute the local $\mu$-index $\mu_{j}=\mu\left(\gamma_{j}, \tilde{\xi}, \Phi_{j}\right)$ of $\gamma_{j}$. We denote by $\mu^{\prime}(\gamma)$ the sum of these local indexes. Unfortunately, $\mu^{\prime}(\gamma)$ is not the Conley-Zehnder index that we want because the local trivializations of $\tilde{\xi}$ by $\Phi_{j}$ do not match up to a symplectic trivialization of $\left.\tilde{\xi}\right|_{D}$. There are two types of factors which cause mismatches of these local trivializations:

(1) The choices of a framing of the normal bundle of the attaching isotropic spheres.

(2) The gluing of a $k$-handle (using the flow of $Y_{\text {st }}$ ).

Type 1 can be overcome by choosing suitable c's (to produce large $N_{1}(\mathbf{c})$ ). Type 2 happens each time $\gamma$ crosses from one $\mathcal{H}(k)$ to another. The gluing map $\eta$ preserves contact structure $\xi$ but not contact forms. Let $\alpha^{\prime}:=\eta^{*} \alpha=e^{-h} \alpha$, 
and let $R$ denote the Reeb vector field of $\alpha$, then the Reeb vector field $R^{\prime}$ of $\alpha^{\prime}$ is

$$
R^{\prime}=e^{h}\left(R+X_{h}^{\xi}\right)
$$

where $X^{\xi}$ is the vector field tangent to $\xi$ and satisfying

$$
d \alpha\left(X_{h}^{\xi}, \cdot\right)=-\left.d h\right|_{\xi}
$$

Since the actual gluing take place in a thin collar of $\partial U_{-}$, we may assume that $h \sim$ const and $R^{\prime} \sim e^{h} R$ on the collar. Then by mimicking the comparison of Hamiltonian flow and Reeb flow in the previous section, we conclude that each Type 2 error is bounded by $\pm 2 n$.

Let $\tau_{j}$ be the action of $\gamma_{j}$ and $\tau=\sum_{j=1}^{m-1} \tau_{j}$ be the action of $\gamma$. By Lemma 4.4 we have

$$
\tau>C_{m} T
$$

which together with (13) shows

$$
\mu^{\prime}(\gamma)>\sum_{j=1}^{m-1} N_{1}(\mathbf{c}) \cdot \tau_{j}+N_{2}(\mathbf{c}) \cdot C_{m} T-2 n m .
$$

Then the actual Conley-Zehnder index $\mu(\gamma)=\mu(\gamma, \tilde{\xi}, D)$ satisfies the inequality

$$
\mu(\gamma)>\left(\sum_{j=1}^{m-1} N_{1}(\mathbf{c}) \cdot \tau_{j}-(\text { error of Type } 1)\right)+N_{2}(\mathbf{c}) \cdot C_{m} T-4 m n
$$

The first term on the right hand side of (14) can be made positive and very large by choosing suitable $c_{l}$ as discussed before. For the second term, recall $C_{m}=\max \left\{1,\left[\frac{m}{2^{n}}\right]\right\}$, then $N_{2}(\mathbf{c}) \cdot C_{m} T-4 m n$ can be very large if we choose to have $N_{2}(\mathbf{c}) \gg 2^{n}$ (by choosing suitable $c_{l}$ ) and $T \gg 4 n$. Note that none of these $N_{1}(\mathbf{c}), N_{2}(\mathbf{c})$ and $T$ depend on $m$ or on $\gamma$. This completes the proof of Lemma 4.2

\section{$5 \quad$ Stabilization of $(M, \xi)$}

Let $(M, \xi)$ be a $(2 n-1)$-dimensional contact manifold. Let $(W, \omega, Y, f)$ be an Weinstein manifold associated to $(M, \xi)$ as discussed in the previous section. We many assume that $M=\{f=c\}$ for some suitable constant $c$. We also assume that $\operatorname{dim} M>3$ for the moment. This condition on dimension is to ensure that $\partial \circ \partial=0$ because $C_{*}(M, \alpha)=0$ for $* \leq 1$ when $\operatorname{dim} M>3$. Later 
we will show that $\partial \circ \partial=0$ also holds true when $\operatorname{dim} M=3$ despite of the fact that $C_{1}(M, \alpha) \neq 0$ when $\operatorname{dim} M=3$.

By now we have seen that simple Type I Reeb orbits of a SSFC manifold $(M, \xi)$ are in one-one correspondence with critical points of $f$ on $W$. One might expect that the counting of 1-dimensional moduli of holomorphic cylinders here is equivalent to the counting of gradient trajectories of $f$ connecting critical points of consecutive Morse indexes. Let $p \in \operatorname{Crit}(f)$ be of index $k<n$ $(2 n=\operatorname{dim} W)$, and let

$$
S_{c}(p) \cong\{\mathrm{f}=c\} \cap\{|y|=0\} \quad(\text { see (4) })
$$

be the $(2 n-k-1)$-dimensional coisotropic ellipsoid in the corresponding contact $k$-handle. We may identify $\gamma_{p}$ with $\left\{\left|z_{n}\right|^{2}=c_{n}^{2} c\right\} \cap S_{c}(p)$. $S^{1}$ acts on $S_{c}(p)$ by rotating the $z_{n}$-plane, giving $S_{c}(p)$ an open book structure with binding $B:=S_{c}(p) \cap\left\{z_{n}=0\right\} \cong S^{2 n-k-3}$, pages diffeomorphic to a $(2 n-k-2)-$ dimensional disc $D^{2 n-k-2}$, and $S_{c}(p) \backslash B \cong S^{1} \times D^{2 n-k-2}$. Then by following the discussion in Section $\left[7\right.$ on $S^{1}$-invariant holomorphic curves, one can see that $S_{c}(p) \backslash \gamma_{p}$ is foliated by 2-dimensional discs bounding $\gamma_{p}$ and all such discs are images of some element of $\mathcal{M}\left(\gamma_{p}\right) / \mathbb{R}$ before contact handles of higher indexes are attached. In particular $B$ is the parameter space of a connected component of $\mathcal{M}\left(\gamma_{p}\right) / \mathbb{R}$.

Suppose now that a contact $(k+1)$-handle (assuming $k+1<n)$ corresponding to $q \in C r i t(f)$ is attached along an isotropic $k$-sphere which intersects transversally with $S_{c}(p) \backslash \gamma_{p}$ at finitely many points. Assume that these intersection points are on distinct elements of $\mathcal{M}\left(S_{c}(p) ; \gamma_{p}\right) / \mathbb{R}$. Intuition suggests these "marked" (by the intersection points) elements in $\mathcal{M}\left(S_{c}(p) ; \gamma_{p}\right) / \mathbb{R}$ may correspond to elements in $\mathcal{M}\left(\gamma_{q}, \gamma_{p}\right) / \mathbb{R}$ of the resulting manifold. This is where we get the speculation that perhaps the counting of holomorphic cylinders is equivalent to the counting of gradient trajectories. Of course many works have to be done to verify (or disprove) such a naive speculation.

On the other hand, if the above guess is true for $(M, \xi)$ viewed as a regular level set of a subcritical Weinstein manifold $(W, \omega, Y, f)$, then it is also true for $\left(M^{\prime}, \xi^{\prime}\right)$ which is the corresponding regular level set of $f+\kappa|z|^{2}: W \times \mathbb{C} \rightarrow \mathbb{R}$ with $\kappa>0$. It turns out that $\left(M^{\prime}, \xi^{\prime}\right)$ has several nice features which allow an alternative approach of computing $H C_{*}(M, \xi)$ and establishing a relation between $H C_{*}(M, \xi)$ and $H_{*}(W)$ as one has expected. The rest of this section consists of more discussion on $\left(M^{\prime}, \xi^{\prime}\right)$, which serves as preparation for the next two sections.

Recall that from $(W, \omega, Y, f)$ we can define a new Weinstein manifold

$$
\left(W^{\prime}, \omega^{\prime}, Y^{\prime}, f^{\prime}\right):=\left(W \times \mathbb{C}, \omega+\omega_{o}, Y+Y_{o}, f+\kappa|z|^{2}\right)
$$


where $\omega_{o}=d x \wedge d y$ is the standard symplectic structure on $\mathbb{C} ; Y_{o}:=\frac{1}{2}\left(x \partial_{x}+\right.$ $\left.y \partial_{y}\right)$ is a Liouville vector field with respect to $\omega_{o}$; and $\kappa>0$ is a constant. Here $z=x+i y$ is the complex coordinate of $\mathbb{C} .\left(W^{\prime}, \omega^{\prime}, Y^{\prime}, f^{\prime}\right)$ is called a stabilization of $(W, \omega, Y, f)$.

Consider on $W^{\prime}$ the hypersurface

$$
M^{\prime}:=\left\{f^{\prime}=c\right\} .
$$

$M^{\prime}$ is a regular level set of $f^{\prime}$. The 1 -form $\alpha^{\prime}:=\omega^{\prime}\left(Y^{\prime}, \cdot\right)$ restricts to a contact 1-form on $M^{\prime}$. Denote by $\xi^{\prime}$ the associated contact structure on $M^{\prime}$. It is easy to see that $(M, \xi)$ is a codimension 2 contact submanifold of $\left(M^{\prime}, \xi^{\prime}\right)$. If $M$ is subcritical, then so is $M^{\prime}$. Note that the rotation in $\mathbb{C}$ centered at $z=0$ induces an $S^{1}$-action on $M^{\prime}$ that acts freely on $M^{\prime} \backslash M$ and fixes $M$. Indeed, we can view $M^{\prime}$ as an open book with binding $M$, pages diffeomorphic to $V$, and trivial monodromy $i d: V \rightarrow V$. Here $V:=\{f<c\} \subset W$ is called a subcritical Stein-filling of $M$.

We can smoothly embed $\mathbb{R} \times M^{\prime}$ into $W^{\prime}$ by identifying $\{0\} \times M^{\prime}$ with $M^{\prime} \subset$ $W^{\prime}$, and the vector field $\frac{\partial}{\partial t}$ with $Y^{\prime}$. The image of $\mathbb{R} \times M^{\prime}$ in $W^{\prime}$ is then $W_{o}^{\prime}:=W^{\prime} \backslash \mathcal{L}$, where $\mathcal{L}$ is the closure of the stable submanifolds of the flow of $Y^{\prime}$. The image of $\mathbb{R} \times M$ is $W_{o}:=W \backslash \mathcal{L}$.

Lemma 5.1 $\left.c_{1}(\xi ; M)\right|_{\pi_{2}(M)}=0$ if and only if $\left.c_{1}\left(\xi^{\prime} ; M^{\prime}\right)\right|_{\pi_{2}\left(M^{\prime}\right)}=0$.

Proof Let $\iota: M \hookrightarrow M^{\prime}$ be the inclusion map and $S$ represent an element of $\pi_{2}\left(M^{\prime}\right)$. Since $M^{\prime} \cong M \times D^{2} \cup_{M \times S^{1}} V \times S^{1}, \operatorname{dim} V=2 n \geq 4$ and $H_{2 n-2}(W)=$ $0, S$ can be pushed into $M$, ie, $S$ represents an element of $\pi_{2}(M)$. On the other hand an element of $\pi_{2}(M)$ is also an element of $\pi_{2}\left(M^{\prime}\right)$. Since $\iota^{*} c_{1}\left(\xi^{\prime}\right)=c_{1}(\xi)$ we conclude that $\left.c_{1}(\xi ; M)\right|_{\pi_{2}(M)}=\left.0 \Leftrightarrow c_{1}\left(\xi^{\prime} ; M^{\prime}\right)\right|_{\pi_{2}\left(M^{\prime}\right)}=0$.

Although $\alpha^{\prime}$ may not be regular in the usual sense ( $\alpha^{\prime}$ may have $S^{1}$-families of Reeb orbits), the above properties ensure that cylindrical contact homology of $\left(\xi^{\prime}, \alpha^{\prime}\right)$ is still defined.

The Reeb vector field of $\left(M^{\prime}, \alpha^{\prime}\right)$ is

$$
R^{\prime}:=\frac{X_{f}+4 \kappa i Y_{o}}{\alpha\left(X_{f}\right)+\kappa|z|^{2}}
$$

We may assume that each critical point of $f$ is standard, then so are the critical points of $f^{\prime}$ (note that $f$ and $f^{\prime}$ have the same set of critical points with the same Morse indexes). Since there are only finitely many critical points, $\kappa$ can 
be chosen so that $\left\{c_{k+1}^{2}, \ldots, c_{n}^{2}, \kappa^{-1}\right\}$ is linearly independent over $\mathbb{Q}$ for any $\left\{c_{k+1}^{2}, \ldots, c_{n}^{2}\right\}$ associated to some critical point of index $k$ of $f$.

When $\kappa$ is much smaller than any of those $c_{j}$ then the principal Reeb orbits of $\left(M^{\prime}, \alpha^{\prime}\right)$ are exactly principal Reeb orbits of $(M, \alpha)$. On the other hand, if $\kappa$ is much bigger than any of those $c_{l}$ then the simple principal Reeb orbits of $\left(M^{\prime}, \alpha^{\prime}\right)$ are

$$
\gamma_{p}:=\left\{(p, z) \in M^{\prime} \mid p \in \operatorname{Crit}(f)\right\}
$$

they are in one-one correspondence with critical points of $f$. Certainly the contact homology of $\left(M^{\prime}, \xi^{\prime}\right)$ does not depend on the choice of $\kappa$. In fact, we will show that, up to a degree shifting by $2, H C(M, \xi)$ and $H C\left(M^{\prime}, \xi^{\prime}\right)$ are isomorphic. Thus we know about $H C_{*}(M, \xi)$ once we know about $H C_{*}\left(M^{\prime}, \xi^{\prime}\right)$.

\section{$6 \quad H C_{*}(M, \xi)=H C_{*+2}\left(M^{\prime}, \xi^{\prime}\right)$}

Recall that $Y_{o}=\frac{1}{2}\left(x \partial_{x}+y \partial_{y}\right)$. Let $Y \subset T W$ be a gradient-like vector field with respect to $f$ and let $b>0$ be a constant. The vector field $Y^{\prime}:=Y+b Y_{o} \subset T W^{\prime}$ is gradient-like with repsect to $f^{\prime}$. Let $Y^{\prime t}$ denote the time $t$ map of the flow of $Y^{\prime}$. We can embed $\mathbb{R} \times M^{\prime}$ into $W^{\prime}$ by identifying $\{t\} \times M^{\prime} \subset \mathbb{R} \times M^{\prime}$ with $Y^{\prime t}\left(M^{\prime}\right) \subset W^{\prime}$. In particular the vector field $\partial_{t} \subset T\left(\mathbb{R} \times M^{\prime}\right)$ is identified with $Y^{\prime}$. Note that $\mathbb{R} \times M \subset W \times\{0\}$ under this identification.

We need to know how to count pseudo-holomorphic curves in $\operatorname{Symp}(M)=$ $\mathbb{R} \times M$ and $\operatorname{Symp}\left(M^{\prime}\right)=\mathbb{R} \times M^{\prime}$. To achieve our goal, we first choose a class of admissible almost complex structures. First of all observe that the group $S^{1}$ acts on $W \times \mathbb{C}$ by rotations on $\mathbb{C}$, sending $(p, z)$ to $\left(p, e^{i \theta} z\right)$ for $\theta \in S^{1} \cong \mathbb{R} /(2 \pi \mathbb{Z})$. It restricts to an $S^{1}$-action on $M^{\prime}$ that fixes $M$, acts freely on $M^{\prime} \backslash M$, and preserves $\alpha^{\prime}$. Let $\Pi_{\mathbb{C}}$ denote the projection $W \times \mathbb{C} \rightarrow \mathbb{C}$, and $\Pi$ the the projection $W \times \mathbb{C} \rightarrow W$. One might expect to find an $\alpha^{\prime}$-admissible almost complex structure which splits and preserves the subbundles $\Pi^{*} T W$ and $\Pi_{\mathbb{C}}^{*} T \mathbb{C}$ of $T W^{\prime}$. This is however, not true in general.

On the other hand, since

$$
\left.\xi^{\prime}\right|_{M}=\xi \oplus \underline{\mathbb{C}}
$$

we consider an $d \alpha^{\prime}$-admissible almost complex structure $J^{\prime}$ on $\xi^{\prime} \subset T M^{\prime}$ such that $J^{\prime}$ preserves the decomposition (15), and $J^{\prime}=i$ when restricted to the second factor of (15), here $i$ denote the standard complex structure on $\mathbb{C}$. It is easy to see that there are plenty of $d \alpha^{\prime}$-compatible almost complex structures satisfying the above condition. Then we extend $J^{\prime}$ to become an admissible 
almost complex structure on $\operatorname{Symp}\left(M^{\prime}\right) \subset W^{\prime}$ via the flow of $Y^{\prime t}$. In particular, $J^{\prime}\left(Y^{\prime}\right)=Y_{*}^{\prime t} R^{\prime}$ on $Y^{\prime t}\left(M^{\prime}\right)$. We have

$$
J^{\prime}=\left[\begin{array}{cc}
J & O \\
O & i
\end{array}\right]+Q, \quad Q \rightarrow O \text { as }|z| \rightarrow 0, z \in \mathbb{C} .
$$

Theorem 6.1 $H C_{*}(M, \xi ; \alpha, J) \cong H C_{*+2}\left(M^{\prime}, \xi^{\prime} ; \alpha^{\prime}, J^{\prime}\right)$.

Proof Fix any large positive integer $m_{o} . M$ can be constructed as a level set of the Weinstein manifold $(W, \omega, Y, f)$ such that the following action condition is satisfied: Let $\gamma_{p}^{m}$ denote the principal generator corresponding to the critical point $p$ of $f$ and with multiplicity $m$. Then $\mathcal{A}\left(\gamma_{p}^{1}\right)>\mathcal{A}\left(\gamma_{q}^{m}\right)$ when the index of $p$ is greater than the index of $q$ and $m \leq m_{o}$.

Let $\kappa$ be small enough then the principal generators of the contact complex $C_{*}(M, \alpha)$ are principal generators of $C_{*}\left(M^{\prime}, \alpha^{\prime}\right)$. Let $\gamma_{+}$and $\gamma_{-}$be two such principal generators, then

$$
\mathcal{M}:=\mathcal{M}_{J}^{\prime}\left(M ; \gamma_{-}, \gamma_{+}\right) \subset \mathcal{M}^{\prime}:=\mathcal{M}_{J^{\prime}}\left(M^{\prime} ; \gamma_{-}, \gamma_{+}\right) .
$$

Given $u^{\prime} \in \mathcal{M}^{\prime}$, write $u^{\prime}=\left(u_{1}, u_{2}\right)$ according to the splitting $W^{\prime}=W \times \mathbb{C}$. Assume $u^{\prime} \notin \mathcal{M}$, then $u_{2} \not \equiv 0$. The $u_{2}$-component of $u^{\prime}$ associates two winding numbers (recall that $\operatorname{Symp}(M) \subset W$ )

$$
\begin{aligned}
& n_{-}:=\operatorname{wind}\left(u^{\prime} \cap\{t \ll 0\}, \operatorname{Symp}(M)\right), \\
& n_{+}:=\operatorname{wind}\left(u^{\prime} \cap\{t \gg 0\}, \operatorname{Symp}(M)\right) .
\end{aligned}
$$

Since $u^{\prime}\left(\mathbb{C}^{*}\right)$ and $\operatorname{Symp}(M)$ are pseudo-holomorphic submanifolds of complement dimensions, $u^{\prime}\left(\mathbb{C}^{*}\right)$ intersects with $\operatorname{Symp}(M)$ positively at every point of the intersection $u^{\prime}\left(\mathbb{C}^{*}\right) \cap \operatorname{Symp}(M)$. Thus we have

$$
n_{+}-n_{-}=\#\left(u^{\prime}\left(\mathbb{C}^{*}\right) \cap \operatorname{Symp}(M)\right) \geq 0 .
$$

Write $u_{2}=u_{2}(w)$ where $w$ denotes the complex coordinate of $\mathbb{C} . u_{2}: \mathbb{C}^{*} \rightarrow \mathbb{C}$ is a smooth function. Recall that we embed $\operatorname{Symp}\left(M^{\prime}\right)=\mathbb{R} \times M^{\prime}$ into $W^{\prime}=$ $W \times \mathbb{C}$ by identifying $\partial_{t}$ with $Y^{\prime}=Y+b Y_{o}$ for some constant $b>0$. The integral trajectories of $Y^{\prime}$ perserves the value $|z|^{-b / 2}$. Since $u^{\prime}(w)$ approaches $\operatorname{Symp}(M) \subset W \times\{0\}$ asymptotically as $w$ approaches either 0 or $\infty$ we have

$$
\begin{gathered}
u_{2}(w) \text { is asymptotically holomorphic as }|w| \rightarrow 0 \text { or } \infty, \\
\left|u_{2}(w)\right|^{-b / 2} \rightarrow 0 \quad \text { as }|w| \rightarrow 0 \text { or } \infty .
\end{gathered}
$$

$u_{2}$ can be continuously extended to $\mathbb{C}$ by defining $u_{2}(0):=0$. The extended function is still denoted by $u_{2}$ for simplicity. 
Now by (18) and (19) we have that near $w=0$,

$$
u_{2}(w) \sim w^{n_{-}} \quad \text { for some } n_{-} \in \mathbb{N}, n_{-}>b / 2 .
$$

Here $n_{-}$is exactly the earlier defined winding number of $u^{\prime}\left(\mathbb{C}^{*}\right)$ to $\operatorname{Symp}(M)$ near $t=-\infty$. Similarly, near $w=\infty$ we have

$$
u_{2}(w) \sim w^{n_{+}} \quad \text { for some } n_{+} \in \mathbb{N}, n_{+}<b / 2,
$$

where $n_{+}$is the winding number of $u^{\prime}\left(\mathbb{C}^{*}\right)$ to $\operatorname{Symp}(M)$ near $t=\infty$. Then we have $n_{+}<b / 2<n_{-}$and in particular $n_{+}-n_{-}<0$, which contradicts with (17). So $u_{2} \equiv 0$. We conclude that $\mathcal{M}=\mathcal{M}^{\prime}$. The degree 2 shift is an easy observation. This completes the proof.

\section{$7 \quad$ Finding $H C(M, \xi)$}

We now proceed to compute the cylindrical contact homology of $\left(M^{\prime}, \xi^{\prime}\right)$ (again we assume that $\operatorname{dim} M>3$ ). This time we choose to have $\kappa \gg 1$ so that the principal Reeb orbits are in $M^{\prime} \backslash M$ and they are in one-one correspondence with elements of $C r i t(f)$. More precisely they are

$$
\gamma_{p}:=\{(p, z)\} \subset M^{\prime}, \quad p \in \operatorname{Crit}(f)
$$

with index $\bar{\gamma}_{p}=2 n-\operatorname{ind}(p)$.

Let $J^{\prime}$ be the same as in the previous section. To determine the boundary operator of the contact complex we first need to characterize all moduli of the form $\mathcal{M}^{\prime}\left(\gamma_{-}, \gamma_{+}\right)$of formal dimension equal to 1 .

Lemma 7.1 Fix $m_{o}>0$ then there are contact 1 -forms on $\left(M^{\prime}, \xi^{\prime}\right)$ with nondegenerate Type I Reeb orbits such that if are of Type I with multiplicity $\leq m_{o}$ and if the formal dimension of $\mathcal{M}_{J^{\prime}}^{\prime}\left(\gamma_{-}, \gamma_{+}\right)$is 1 then $\gamma_{ \pm}=\gamma_{p_{ \pm}}^{m}$ for some $p_{ \pm} \in \operatorname{Crit}(f)$ with ind $\left(p_{-}\right)=\operatorname{ind}\left(p_{+}\right)+1$ and $\gamma_{ \pm}$have the same multiplicity $m$.

Proof Let $\mathcal{M}^{\prime}=\mathcal{M}_{J^{\prime}}^{\prime}\left(\gamma_{-}, \gamma_{+}\right)$be nonempty and its formal dimension is $\bar{\gamma}_{+}-$ $\bar{\gamma}_{-}=1 . \quad \gamma_{+}=\gamma_{p_{+}}^{m_{+}}$for some $p_{+} \in \operatorname{Crit}(f)$ with $\operatorname{ind}\left(p_{+}\right)=k_{+}, \gamma_{-}=\gamma_{p_{-}}^{m_{-}}$, where $\gamma_{p_{-}}, m_{-}$, and $k_{-}$are defined similarly, and $m_{ \pm} \leq m_{o}$. We have

$$
\begin{aligned}
1 & =2 n-k_{+}+2\left(m_{+}-1\right)-\left(2 n-k_{-}+2\left(m_{-}-1\right)\right) \\
& =k_{-}-k_{+}+2\left(m_{+}-m_{-}\right),
\end{aligned}
$$

therefore $k_{-} \neq k_{+}$. If $k_{-}<k_{+}$then $\mathcal{A}\left(\gamma_{+}\right)<\mathcal{A}\left(\gamma_{-}\right)$which is impossible, so $k_{-}>k_{+}$. 
Assume $k_{-}>k_{+}+1$ then $m_{+}<m_{-}$. Note that $m_{ \pm}=n_{ \pm}$are the winding numbers of $u^{\prime}$ around $\operatorname{Symp}(M)=R \times M$ near $t= \pm \infty$ respectively. Since $n_{+} \geq n_{-}$it cannot happen that $k_{-}>k_{+}+1$. So we must have $k_{-}=k_{+}+1$ and hence $m_{+}=m_{-}$.

By using $n_{+}-n_{-}=\#\left(u^{\prime}\left(\mathbb{C}^{*}\right) \cap \operatorname{Symp}(M)\right)$ and the positivity of the intersection $u^{\prime}\left(\mathbb{C}^{*}\right) \cap \operatorname{Symp}(M)$ we have the following simple lemma:

Lemma 7.2 Let $\mathcal{M}^{\prime}=\mathcal{M}_{J^{\prime}}^{\prime}\left(\gamma_{-}, \gamma_{+}\right)$be as in the previous lemma and let $u^{\prime} \in \mathcal{M}^{\prime}$. Then the curve $u^{\prime}\left(\mathbb{C}^{*}\right)$ does not intersect $\mathbb{R} \times M$.

Since all curves that we are going to count are in the symplectization of $M^{\prime} \backslash M$ we can use the diffeomorphism $M^{\prime} \backslash M \cong V \times S^{1}$ to simplify the computation.

Consider the diffeomorphism :

$$
\Phi: V \times S^{1} \rightarrow M^{\prime} \backslash M, \quad \Phi(x, \theta)=\left(x, \sqrt{\frac{c-f}{\kappa}} \theta\right)
$$

Then $\Phi^{*}\left(\alpha^{\prime}\right)=e^{-h} d \theta+\alpha$. Write $\Phi^{*}\left(\alpha^{\prime}\right)=e^{-h}\left(d \theta+e^{h} \alpha\right)$ then by using the fact that $e^{-h}\left(d \theta+e^{h} \alpha\right)$ is contact one sees that $d\left(e^{h} \alpha\right)$ is symplectic on $V$. So by abusing notations we redenote $e^{h} \alpha$ as $\alpha$ and denote $\lambda:=d \theta+\alpha$.

$\lambda$ is a connection 1 -form on the trivial principal bundle

$$
\begin{gathered}
V \times S^{1} \stackrel{\pi}{\rightarrow} V, \\
d \lambda=\pi^{*} \omega, \quad \omega:=d \alpha
\end{gathered}
$$

and $h$ is a smooth Morse function on $V$ with $\operatorname{Crit}(h)=\operatorname{Crit}(f)$ and the same corresponding Morse indexes.

With the above isomorphism understood we will from now on work with the contact manifold $\left(V \times S^{1}, e^{-h} \lambda\right)$. We denote the corresponding contact structure by $\xi^{\prime}$. $\xi^{\prime}$ is the horizontal lifting of $T V$ with respect to the connection 1-form $\lambda$. Let $X_{h}$ be the Hamiltonian vector field of $h$ with respect to the symplectic $2-$ form $\omega$, ie, $\omega\left(X_{h}, \cdot\right)=-d h$. Then the Reeb vector field of $e^{-h} \lambda$ is

$$
R^{\prime}=\frac{e^{h}}{1+\alpha\left(X_{h}\right)}\left(\partial_{\theta}+X_{h}\right)
$$

with $1+\alpha\left(X_{h}\right)>0$ on $V \times S^{1}$.

$S^{1}$ acts freely on $V \times S^{1}$ by rotation along $S^{1}$ fibers. Let $J^{\prime}$ be an $S^{1}$-invariant $e^{-h} \lambda$-admissible almost complex structure. Since $\xi^{\prime}$ is transversal to the fibers, $J_{\xi^{\prime}}:=\left.J^{\prime}\right|_{\xi^{\prime}}$ induces an $\omega$-compatible almost complex structure $\bar{J}$ on $V$ by

$$
\bar{J}\left(\pi_{*} \eta\right):=\pi_{*} J^{\prime} \eta \quad \eta \in \xi^{\prime} .
$$


Conversely an $\omega$-compatible almost complex $\bar{J}$ structure on $V$ induces an $S^{1}$-invariant $d \lambda$-compatible almost complex structure $J_{\xi^{\prime}}$ on $\xi^{\prime}$ which extends to be an $S^{1}$-invariant $e^{-h} \lambda$-admissible almost complex structure $J^{\prime}$ on the symplectization of $V \times S^{1}$.

Let $\gamma_{ \pm}=\gamma_{p_{ \pm}}^{m}$ for some $p_{ \pm} \in \operatorname{Crit}(h)$ and $u^{\prime} \in \mathcal{M}_{J^{\prime}}\left(\gamma_{-}, \gamma_{+}\right)$be with $S^{1-}$ invariant image. Let

$$
u=(\bar{u}, \theta): \mathbb{R} \times S^{1} \rightarrow V \times S^{1}
$$

be the corresponding map into $V \times S^{1}$. Since $C:=u\left(\mathbb{R} \times S^{1}\right)$ is $S^{1}$-invariant and $\xi^{\prime}$ is transversal to the $S^{1}$-fibers, $\xi^{\prime}$ induces a nonsingular foliation on $C$ generated by $\xi^{\prime} \cap T C$. We can reparametrize $u^{\prime}$ so that

$$
\lambda\left(u_{s}\right)=0, \quad \lambda\left(u_{t}\right)=m .
$$

Here $(s, t)$ are coordinates for $\mathbb{R} \times S^{1}$ (so $z=s+i t$ is the complex coordinate), and $u_{s}:=\frac{\partial u}{\partial s}, u_{t}:=\frac{\partial u}{\partial t}$.

Let $\pi_{1}: T\left(V \times S^{1}\right) \rightarrow \xi^{\prime}$ be the projection along the Reeb vector field $R^{\prime}$. Since $u^{\prime}$ is $J^{\prime}$-holomorphic we have

$$
\pi_{1} u_{s}+J^{\prime} \pi_{1} u_{t}=0
$$

ie,

$$
u_{s}-\frac{\lambda\left(u_{s}\right)}{1+\alpha\left(X_{h}\right)}\left(\partial_{\theta}+X_{h}\right)+J^{\prime}\left(u_{t}-\frac{\lambda\left(u_{t}\right)}{1+\alpha\left(X_{h}\right)}\left(\partial_{\theta}+X_{h}\right)\right)=0,
$$

which by (20) is reduced to

$$
u_{s}+J^{\prime}\left(u_{t}-m \rho\left(\partial_{\theta}+X_{h}\right)\right)=0,
$$

where $\rho=\left(1+\alpha\left(X_{h}\right)\right)^{-1}$. Write $u_{s}=\left(\bar{u}_{s}, \theta_{s}\right), u_{t}=\left(\bar{u}_{t}, \theta_{t}\right)$. Apply $\pi_{*}$ to (22) and we have

$$
\bar{u}_{s}+\bar{J}\left(\bar{u}_{t}-m \rho X_{h}\right)=0 .
$$

Hence $\bar{u}$ is a finite-energy solution to the Cauchy-Riemann type equation as in Floer Theory with

$$
\bar{u}_{s}=m \bar{J}\left(\rho X_{h}\right), \quad \bar{u}_{t}=0 .
$$

Note that the flow of the vector field $m \rho \bar{J} X_{h}$ is of Morse-Smale type for generic $\bar{J}$ hence by [31] the linearization of (23) at an $S^{1}$-invariant solution $\bar{u}$

$$
F_{\bar{u}} \bar{\eta}=\bar{\nabla}_{s} \bar{\eta}+\bar{J} \bar{\nabla}_{t} \bar{\eta}-m \bar{\nabla}_{\bar{\eta}}\left(\bar{J}\left(\rho X_{h}\right)\right)
$$

is onto for generic $\bar{J}$. Here $\bar{\nabla}$ is the Levi-Civita connection associated to the Riemannian metric $\bar{g}:=d \alpha \circ(I d \times \bar{J})$. 
Conversely an $S^{1}$-invariant solution $\bar{u}$ to (23) can be "lifted" to an $S^{1}$-invariant $J^{\prime}$-holomorphic map $u^{\prime}=(a, u)$ into $\operatorname{Symp}\left(V \times S^{1}\right)$ as follows. First we lift $\bar{u}$ to a map $u=(\bar{u}, \theta)$ into $V \times S^{1}$ with $\theta=\theta(s, t): \mathbb{R} \times S^{1} \rightarrow S^{1}$ satisfying

$$
\theta_{s}=-\alpha\left(\bar{u}_{s}\right), \quad \theta_{t}=m-\alpha\left(\bar{u}_{t}\right)=m .
$$

Such $\theta$ exists and is unique up to the addition of a constant rotation. The resulting map $u$ satisfies (21) and (20).

Now solve for the function $a=a(s, t): \mathbb{R} \times S^{1} \rightarrow \mathbb{R}$ which satisfies

$$
a_{s}=e^{-h} \lambda\left(u_{t}\right)=m e^{-h}, \quad a_{t}=-e^{-h} \lambda\left(u_{s}\right)=0 .
$$

Since $\mathbb{R} \times S^{1}$ is a noncompact Riemann surface, there exists a complex-valued function $b: \mathbb{R} \times S^{1} \rightarrow \mathbb{C}$ such that

$$
b_{s}=e^{-h} \lambda\left(u_{t}\right)=m e^{-h}, \quad b_{t}=-e^{-h} \lambda\left(u_{s}\right)=0 .
$$

$b$ is unique up to an addition of a holomorphic function. Write $b_{1}$ for the real part of $b$, and $b_{2}$ for the imaginary part of $b$. We have

$$
\left(b_{2}\right)_{s s}+\left(b_{2}\right)_{t t}=-u_{s}\left(e^{-h} \lambda\left(u_{s}\right)\right)-u_{t}\left(e^{-h} \lambda\left(u_{t}\right)\right)=0
$$

ie, $b_{2}$ is harmonic, hence the imaginary part of a holomorphic function $\tilde{b}$. Define $a:=b-\tilde{b} . \quad a$ is a real-valued function on $\mathbb{R} \times S^{1}$ and satisfies (25). Moreover $a$ is unique up to the addition of a real constant. The resulting map $u^{\prime}$ is $S^{1}$-invariant, $J^{\prime}$-holomorphic with multiplicity $m$, unique up to the rotation by a constant angle and the addition of a real constant and satisfies

$$
\begin{gathered}
u(s, \cdot) \rightarrow \gamma_{ \pm} \text {as } s \rightarrow \pm \infty, \\
0<\int_{\mathbb{R} \times S^{1}} u^{*} d\left(e^{-h} \lambda\right)=\int_{\gamma_{+}} e^{-h} \lambda-\int_{\gamma_{-}} e^{-h} \lambda=m\left(e^{-h\left(p_{+}\right)}-e^{-h\left(p_{-}\right)}\right)<\infty .
\end{gathered}
$$

Hence $u^{\prime} \in \mathcal{M}_{J^{\prime}}^{\prime}\left(\gamma_{-}, \gamma_{+}\right)$.

Now that we have establishes for each fixed $m \in \mathbb{N}$ (and $m \leq m_{o}$ for some large $m_{o}$ ) a one-one correspondence between (i) the (gradient-like) $\bar{J} X_{h}$-trajectories in $V$ that connecting critical points $p_{ \pm}$with $\operatorname{ind}\left(p_{+}\right)=\operatorname{ind}\left(p_{-}\right)=1$ and (ii) the $S^{1}$-invariant elements in the moduli $\mathcal{M}_{J^{\prime}}^{\prime}\left(\gamma_{-}, \gamma_{+}\right) / \mathbb{R}$ with $\gamma_{ \pm}=\gamma_{p_{ \pm}}^{m}$. We proceed to show that the linearization of the operator $\bar{\partial}:=\bar{\partial}_{J^{\prime}}$ at an $S^{1}$ invariant solution $u^{\prime} \in \mathcal{M}_{J^{\prime}}^{\prime}\left(\gamma_{-}, \gamma_{+}\right)$is surjective for generic $J^{\prime}$.

Recall that

$$
\bar{\partial}\left(u^{\prime}\right)=u_{s}^{\prime}+J^{\prime} u_{t}^{\prime}
$$


Let $\pi_{1}, \pi_{2}$ be the projections with respect to the orthogonal decomposition $T\left(\operatorname{Symp}\left(V \times S^{1}\right)\right) \rightarrow \xi^{\prime} \oplus E, E$ is the vector bundle spanned by $\partial_{t}$ and $R^{\prime}$. Write $\bar{\partial}=\bar{\partial}_{1}+\bar{\partial}_{2}$ where

$$
\begin{aligned}
& \bar{\partial}_{1}\left(u^{\prime}\right)=\pi_{1} u_{s}^{\prime}+J^{\prime} \pi_{1} u_{t}^{\prime}, \\
& \bar{\partial}_{2}\left(u^{\prime}\right)=\pi_{2} u_{s}^{\prime}+J^{\prime} \pi_{2} u_{t}^{\prime} .
\end{aligned}
$$

Let $D_{1}, D_{2}$ denote the linearizations of $\bar{\partial}_{1}$ and $\bar{\partial}_{2}$ at $u^{\prime}$ respectively.

Lemma 7.3 $\pi_{1} D_{1}: W^{1,2}\left(\mathbb{R} \times S^{1}, u^{\prime *} \xi^{\prime}\right) \rightarrow L^{2}\left(\mathbb{R} \times S^{1}, u^{\prime *} \xi^{\prime}\right)$ is surjective for generic $S^{1}$-invariant $J^{\prime}$.

Proof Let $\eta \in W^{1,2}\left(\mathbb{R} \times S^{1}, u^{\prime *} \xi^{\prime}\right)$. Let $\nabla$ be the Levi-Civita connection on $\operatorname{Symp}\left(V \times S^{1}\right)$ with respect to the Riemannian metric $g^{\prime}:=e^{-t+h} d\left(e^{t-h} \lambda\right) \circ$ $\left(I d \times J^{\prime}\right)$. Note that $\left.g^{\prime}\right|_{\xi^{\prime}}=\left.g\right|_{\xi^{\prime}}$ where $g$ is the Riemannian metric on $V \times S^{1}$ induced by $\bar{g}$ and the connection 1 -form $\lambda$ of the $S^{1}$-bundle $V \times S^{1}$ over $V$. Then

$$
\begin{aligned}
D_{1}(\eta)= & \pi_{1} \nabla_{s} \eta-\left(\nabla_{\eta} \lambda\right)\left(u_{s}^{\prime}\right) e^{-h} R^{\prime}+J^{\prime} \pi_{1} \nabla_{t} \eta \\
& +\pi_{1}\left(\nabla_{\eta} J^{\prime}\right) \pi_{1} u_{t}^{\prime}-m J^{\prime} \nabla_{\eta}\left(e^{-h} R^{\prime}\right) .
\end{aligned}
$$

Write $e^{-h} R^{\prime}=\frac{\partial_{\theta}+X_{h}}{1+\alpha\left(X_{h}\right)}=\zeta+\partial_{\theta}$ with $\zeta \in \xi^{\prime}$,

$$
\zeta=\frac{-\alpha\left(X_{h}\right) \partial_{\theta}+X_{h}}{1+\alpha\left(X_{h}\right)}=\frac{-1}{m} \cdot \pi_{1} u_{t}^{\prime}
$$

and apply $\pi_{1}$ to (28) we get

$$
\pi_{1} D_{1}(\eta)=\pi_{1} \nabla_{s} \eta+J^{\prime} \pi_{1} \nabla_{t} \eta-m \pi_{1}\left(\nabla_{\eta} J^{\prime}\right) \zeta-m J^{\prime} \pi_{1}\left(\nabla_{\eta}\left(\zeta+\partial_{\theta}\right)\right) .
$$

Let $\bar{\eta}:=\pi_{*} \eta, \bar{\zeta}:=\pi_{*} \zeta$. Since $\pi_{1}\left(\nabla_{\eta}\left(\left.J^{\prime}\right|_{E}\right)\right) \zeta=0$ and

$$
\begin{aligned}
\pi_{1} \nabla_{\eta} \zeta & =\pi^{*} \bar{\nabla}_{\bar{\eta}} \bar{\zeta} \quad\left(\pi^{*} \text { means "horizontal lifting" }\right) \\
\nabla_{\eta} \partial_{\theta} & =\nabla_{\partial_{\theta}} \eta+\left[\eta, \partial_{\theta}\right]=\nabla_{\partial_{\theta}} \eta-L_{\partial_{\theta}} \eta=0
\end{aligned}
$$

and $\bar{\zeta}=\rho X_{h}$ with $\rho=\left(1+\alpha\left(X_{h}\right)\right)^{-1}$ we have

$$
\begin{aligned}
\pi_{*} \pi_{1} D_{1}(\eta) & =\bar{\nabla}_{s} \bar{\eta}+\bar{J} \bar{\nabla}_{t} \bar{\eta}-m\left(\bar{\nabla}_{\bar{\eta}} \bar{J}\right) \bar{\zeta}-m \bar{J} \bar{\nabla}_{\bar{\eta}} \bar{\zeta} \\
& =\bar{\nabla}_{s} \bar{\eta}+\bar{J} \bar{\nabla}_{t} \bar{\eta}-m \bar{\nabla}_{\bar{\eta}}\left(\bar{J}\left(\rho X_{h}\right)\right) \\
& \left.=F_{\bar{u}}(\bar{\eta}) \quad \text { (see (24) }\right) .
\end{aligned}
$$

Since $F_{\bar{u}}: W^{1,2}\left(\mathbb{R} \times S^{1}, \bar{u}^{*} T V\right)$ is surjective for generic $\bar{J}$ 31] we conclude that $\pi_{1} D_{1}$ is surjective for generic $S^{1}$-invariant $J^{\prime}$. 
Recall that $E$ denote the vector bundle over $\operatorname{Symp}\left(V \times S^{1}\right)$ spanned by $\partial_{t}$ and the Reeb vector field $R^{\prime}$.

Lemma 7.4 $D_{2}: W^{1,2}\left(\mathbb{R} \times S^{1}, u^{\prime *} E\right) \rightarrow L^{2}\left(\mathbb{R} \times S^{1}, u^{\prime *} E\right)$ is surjective for all $J^{\prime}$.

Proof Let $\eta \in W^{1,2}\left(\mathbb{R} \times S^{1}, u^{\prime *} E\right)$ and let $\nabla$ be the Levi-Civita connection defined in proof of Lemma 7.3, Then

$$
D_{2}(\eta)=\pi_{2} \nabla_{s} \eta+J^{\prime} \pi_{2} \nabla_{t} \eta+m\left(\nabla_{\eta} J^{\prime}\right)\left(e^{-h} R^{\prime}\right)+m J^{\prime} \nabla_{\eta}\left(e^{-h} R^{\prime}\right) .
$$

Since $\nabla_{\eta} J^{\prime} \in \operatorname{End}\left(\xi^{\prime}\right), \nabla_{R^{\prime}} R^{\prime}=0, \nabla_{R^{\prime}} e^{-h}=0$ and $\nabla_{\eta}\left(e^{-h} R^{\prime}\right)=0$ we have

$$
D_{2}(\eta)=\pi_{2}\left(\nabla_{s} \eta+J^{\prime} \nabla_{t} \eta\right) .
$$

Write $\eta=\eta_{1} \partial_{t}+\eta_{2} R^{\prime}$ then

$$
D_{2}\left(\left(\eta_{1}, \eta_{2}\right)\right)=\left(\nabla_{s} \eta_{1}-\nabla_{t} \eta_{2}, \nabla_{s} \eta_{2}+\nabla_{t} \eta_{1}\right)
$$

is the standard $d$-bar operator on $W^{1,2}\left(\mathbb{R} \times S^{1}, u^{\prime *} E\right)$ with respect to the almost complex structure $u^{\prime *}\left(\left.J^{\prime}\right|_{E}\right)$ on the trivial complex line bundle $u^{\prime *} E$, hence is surjective because $\mathbb{R} \times S^{1}$ is a noncompact Riemann surface.

Lemma 7.3 and Lemma 7.4 together imply the following:

Lemma 7.5 Let $J^{\prime}$ be an $S^{1}$-invariant $\alpha^{\prime}$-admissible almost complex structure on $\operatorname{Symp}\left(M^{\prime}\right)$. Then for generic $J^{\prime}$ the linearized operator $D=D_{1}+D_{2}$ of $\bar{\partial}_{J^{\prime}}$ is surjective at every $S^{1}$-invariant element of $\mathcal{M}_{J^{\prime}}\left(\gamma_{-}, \gamma_{+}\right)$provided that the multiplicity of $\gamma_{ \pm}$is small.

In the following we would like to show that up to a homotopy of contact 1-forms there are no elements of $\mathcal{M}_{J^{\prime}}\left(\gamma_{-}, \gamma_{+}\right)$which are not $S^{1}$-invariant. Our proof is based on results from 31] concerning finite energy solutions with small periods of Cauchy-Riemann type equations and the following construction.

Recall that the stabilization $M^{\prime}$ can be identified with the following hypersurface in $W \times \mathbb{C}$ :

$$
\left\{f+\kappa|z|^{2}=c\right\}, \quad \kappa \gg 1 \text { fixed, }
$$

with contact structure defined by the 1-form $\alpha^{\prime}=\alpha+\alpha_{o}$, where $\alpha_{o}:=\omega_{o}\left(Y_{o}, \cdot\right)$, $\omega_{o}$ is the standard symplectic 2 -form on $\mathbb{C}$. For each $k \in \mathbb{N}$ with $k>1$ the finite group

$$
\mathbb{Z}_{k}:=\left\{\theta \in S^{1} \mid \theta^{k}=1\right\} \subset S^{1}
$$


acts on $\left(M^{\prime}, \alpha^{\prime}\right)$ via rotation in the $\mathbb{C}$-plane. The action preserves $\alpha^{\prime}$ and induces a branched $k$-covering map

$$
\Phi_{k}: M^{\prime} \rightarrow M^{\prime}
$$

$\Phi_{k}: M^{\prime} \backslash M \rightarrow M^{\prime} \backslash M$ is a $k$-covering map, and $\Phi_{k}$ fixes $M$ pointwise. $\Phi_{k}$ induces a contact 1 -form $\alpha_{k}:=\left(\Phi_{k}\right)_{*} \alpha^{\prime}=\alpha+\alpha_{o} / k$ on $M^{\prime}$. Let $\xi_{k}$ denote the contact structure defined by $\alpha_{k} . \alpha_{k}$ and $\alpha^{\prime}$ can be included into a smooth family of contact 1-forms of $M^{\prime}$ so $\xi_{k}$ and $\xi^{\prime}$ are isotopic as contact structures. Moreover $\left(\Phi_{k}\right)_{*} \xi^{\prime}=\xi_{k}$.

Let $J_{k}$ denote the $S^{1}$-invariant $\alpha_{k}$-admissible almost complex structure on $\operatorname{Symp}\left(V \times S^{1}\right)$ such that

$$
J_{k}=\left(\Phi_{k}\right)_{*} J^{\prime}\left(\Phi_{k}^{-1}\right)_{*} \quad \text { on } \xi_{k} .
$$

Clearly Lemma [7.5] also holds for generic $J_{k}, 1<k \in \mathbb{N}$.

For $v_{k}^{\prime} \in \mathcal{M}_{J_{k}}\left(M^{\prime} ; \gamma_{-}, \gamma_{+}\right)$let $\tilde{v}_{k} \in \mathcal{M}_{J_{k}}\left(\gamma_{-}^{k}, \gamma_{+}^{k}\right)$ be the $k$-fold cover of $v_{k}^{\prime}$. Then $\tilde{v}_{k}\left(s, t+\frac{1}{k}\right)=\tilde{v}_{k}(s, t)$ and the pullback by $\Phi_{k}$ of $\tilde{v}_{k}$ is a $J^{\prime}$-holomorphic $\operatorname{map} u_{k}^{\prime} \in \mathcal{M}_{J^{\prime}}\left(M^{\prime} ; \gamma_{-}, \gamma_{+}\right)$which also satisfies

$$
u_{k}^{\prime}\left(s, t+\frac{1}{k}\right)=\vartheta \cdot u_{k}^{\prime}(s, t),
$$

where $\vartheta$ is the generator of $\mathbb{Z}_{k}$ which represents the $2 \pi / k$-rotation. Conversely if $u_{k}^{\prime} \in \mathcal{M}_{J^{\prime}}\left(M^{\prime} ; \gamma_{-}, \gamma_{+}\right)$satisfies (30) then $\Phi_{k}\left(u_{k}^{\prime}\right) \in \mathcal{M}_{J_{k}}\left(M^{\prime} ; \gamma_{-}^{k}, \gamma_{+}^{k}\right)$ is a $k-$ cover of some $v_{k}^{\prime} \in \mathcal{M}_{J_{k}}\left(M^{\prime} ; \gamma_{-}, \gamma_{+}\right)$.

Lemma 7.6 Assume that ind $\left(p_{-}\right)-\operatorname{ind}\left(p_{+}\right)=1$ and $\gamma_{ \pm}=\gamma_{p_{ \pm}}^{m}$. Then there exists $k_{o} \in \mathbb{N}$ such that for all $k \geq k_{o}$ all elements of $\mathcal{M}_{J_{k}}\left(M^{\prime} ; \gamma_{-}, \gamma_{+}\right)$are $S^{1}$-invariant.

Proof Suppose not. Then there exists an infinite sequence $k_{\nu}$ of positive integers, $\lim _{\nu \rightarrow \infty} k_{\nu}=\infty$, such that for each $k_{\nu}$ the moduli space $\mathcal{M}_{J_{k_{\nu}}}\left(M^{\prime} ; \gamma_{-}, \gamma_{+}\right)$ has an element say $v_{k_{\nu}}^{\prime}$ which is not $S^{1}$-invariant. Let $u_{k_{\nu}}^{\prime}:=\Phi_{k_{\nu}}^{*} \tilde{v}_{k_{\nu}}$ where $\Phi_{k_{\nu}}$ is defined as before, $\tilde{v}_{k_{\nu}}$ is a $k_{\nu}$-cover of $v_{k_{\nu}}^{\prime}$. Then $u_{k_{\nu}}^{\prime} \in \mathcal{M}_{J^{\prime}}\left(M^{\prime} ; \gamma_{-}, \gamma_{+}\right)$. Since all $u_{k_{\nu}}^{\prime}$ have the same contact energy $\mathcal{A}_{\alpha^{\prime}}\left(\gamma_{+}\right)-\mathcal{A}_{\alpha^{\prime}}\left(\gamma_{-}\right)$, there is an infinite subsequence of $u_{k_{\nu}}^{\prime}$, also denoted by $u_{k_{\nu}}^{\prime}$, such that up to translations in $\mathbb{R}$-direction, $u_{k_{\nu}}^{\prime}$ converge to a $J^{\prime}$-holomorphic curve $u^{\prime}$ as $\nu \rightarrow \infty$. $u^{\prime}$ is $S^{1}$-invariant.

All $u_{k_{\nu}}^{\prime}$ and $u^{\prime}$ have the same winding numbers $n_{ \pm}$around $\operatorname{Symp}(M)$ near $t= \pm \infty$. Moreover we have $n_{+}=n_{-}$because none of the $u_{k_{\nu}}^{\prime}$ 's intersect with $\operatorname{Symp}(M)$, so neither does $u^{\prime} . u^{\prime}$ is therefore a finite union of $S^{1}$-invariant 
curves so that the image in $V \times S^{1}$ of each connected component is either a cylinder bounding a pair of type I Reeb orbits. The closure of the image of $u^{\prime}$ in $V$ is a connected tree formed by trajectories of a gradient-like vector field. This tree contains a (perhaps broken) trajectory with endpoints $p_{ \pm}=\pi\left(\gamma_{ \pm}\right)$. Moreover, $p_{ \pm}$are vertices of valent 1 of the tree, here the valent of a vertex is the number of edges coming out from this vertex as an endpoint. Suppose that the this trajectory contains other critical points. Then there is a critical point $p \neq p_{ \pm}$such that a trajectory between $p$ and $p_{+}$is contained in the said broken trajectory from $p_{-}$to $p_{+}$. The corresponding preimage of $p$ in $V \times S^{1}$ is the Reeb orbit $\gamma:=\gamma_{p}^{m}$. Since we must have the action inequalities

$$
\mathcal{A}\left(\gamma_{-}\right)<\mathcal{A}(\gamma)<\mathcal{A}\left(\gamma_{+}\right)
$$

and since $\gamma, \gamma_{-}, \gamma_{+}$have the same multiplicity we have

$$
\operatorname{ind}\left(p_{-}\right)>\operatorname{ind}(p)>\operatorname{ind}\left(p_{+}\right) .
$$

But ind $\left(p_{-}\right)-\operatorname{ind}\left(p_{+}\right)=1$, there exists no such $p$. So the trajectory between $p_{ \pm}$is unbroken and hence is equal to the tree.

Now that the projection of the image of $u^{\prime}$ in $V$ is a trajectory of a gradientlike vector field connecting critical points $p_{-}=\pi\left(\gamma_{-}\right)$to $p_{+}=\pi\left(\gamma_{+}\right)$, so the linearized operator $D_{u^{\prime}}$ is surjective for generic $J^{\prime}$, hence $u^{\prime}$ is an isolated element of $\mathcal{M}_{J^{\prime}}\left(M^{\prime} ; \gamma_{-}, \gamma_{+}\right)$. Thus $u_{k_{\nu}}^{\prime}$ and hence $v_{k_{\nu}}^{\prime}$ have to be $S^{1}$-invariant for all $\nu$ large enough, which contradicts with the assumption that there are non- $S^{1}$-invariant $u_{k_{\nu}}^{\prime}$ for infinitely many $k_{\nu}$. Hence the lemma holds.

Lemma 7.7 Let $\left(\alpha^{\prime}, J^{\prime}\right)$ be a regular pair. For $p \in C r i t(f)$ with $\operatorname{ind}(p)=k$, denote by $\gamma_{p}^{m} \in C_{2(n+m-1)-k}\left(\alpha^{\prime}\right)$ the corresponding principal generator with multiplicity $m \leq m_{o}$. Then

$$
\partial \gamma_{p}^{m}=m \sum_{i n d(q)=k+1} \frac{a_{q}}{m} \gamma_{q}^{m},
$$

where $a_{q}$ is the algebraic number of trajectories of $\bar{J} X_{h}$ running from $q$ and $p$.

Now let $m_{o} \rightarrow \infty$. After an easy computation on index we obtain the following:

Theorem 7.1 Let $\left(M^{\prime}, \xi^{\prime}\right)$ be a stabilization of a $(2 n+1)$-dimensional subcritical Stein-fillable contact manifold $(M, \xi), n>2$, and $(V, J)$ a subcritical Stein-filling of $(M, \xi)$. Then

$$
H C_{i}\left(M^{\prime}, \xi^{\prime}\right) \cong \underset{m \in \mathbb{N} \cup\{0\}}{\oplus} H_{2(n+m)-i}(V) .
$$


Combining Theorem 7.1 with Theorem 6.1 we have the following:

Theorem 7.2 Let $(M, \xi)$ be a $(2 n-1)$-dimensional subcritical Stein-fillable contact manifold with $n>2$, and $V$ a subcritical Stein-filling of $(M, \xi)$. Then

$$
H C_{i}(M, \xi) \cong \underset{m \in \mathbb{N} \cup\{0\}}{\oplus} H_{2(n+m-1)-i}(V) .
$$

When $n=2(M, \xi)$ is the union of $S^{3}$ (3-dimensional contact 0-handle) and a finite number of 3-dimensional contact 1-handles diffeomorphic to $\mathbb{R} \times S^{2}$. We write $s$ for the number of contact 1-handles of $M$. Let $\gamma_{0}$ denote the principal Reeb orbit in the 0 -handle, and $\gamma_{1}, \gamma_{2}, \ldots, \gamma_{s}$ the principal Reeb orbits in each of the $s^{\prime}$ contact 1 -handles. These 1 -handles can be attached to $S^{3}$ pairwise disjoint. Recall that when $n=2 c_{1}(\xi)=0$ so the $\bar{\mu}$-index of contractible Reeb orbits are independent of the spanning discs and hence are well-defined.

Let $m_{o} \gg 1$ be a fixed positive integer, then by deforming 0- and 1-handles we can obtain a suitable regular contact 1 -form $\alpha$ and assume the following:

\section{Condition 7.1}

$$
\begin{aligned}
\overline{\gamma_{j}^{m}} & =\left\{\begin{array}{ll}
2 m & j=0, \\
2 m-1 & j=1,2, \ldots, s
\end{array} \quad \text { for } m \leq m_{o}\right. \\
\mathcal{A}\left(\gamma_{1}\right) & =\mathcal{A}\left(\gamma_{2}\right)=\cdots=\mathcal{A}\left(\gamma_{s}\right) \ll \mathcal{A}\left(\gamma_{0}\right)
\end{aligned}
$$

Let $J$ be a regular $\alpha$-admissible almost complex structure.

Proposition 7.1 Assume $\mathcal{M}:=\mathcal{M}_{J}\left(\gamma_{-}, \gamma_{+}\right)$is not empty and $\bar{\gamma}_{ \pm} \leq 2 m_{o}$.

(1) If $\operatorname{dim} \mathcal{M}=1$ then $\gamma_{-}=\gamma_{j}^{m}, \gamma_{+}=\gamma_{0}^{m}$ for some $m \leq m_{o}, 1 \leq j \leq s$.

(2) If $\operatorname{dim} \mathcal{M}=2$ then $\gamma_{-}=\gamma_{o}^{m-1}, \gamma_{+}=\gamma_{0}^{m}$ for some $m \leq m_{o}$.

Note that $C_{1}(\alpha)$ is nontrivial, it is generated by $\gamma_{j}, 1 \leq j \leq s$. Nevertheless Proposition [7.1 and Condition 7.1 together imply that for $1<m \leq m_{o}$, the boundary of $\mathcal{M}\left(\gamma_{0}^{m-1}, \gamma_{0}^{m}\right)$ does not contain any element of $\mathcal{M}\left(\gamma_{j}^{m}, \gamma_{0}^{m}\right)$. Also when $m=1$ the boundary of $\mathcal{M}\left(\gamma_{0}\right)$, where $\mathcal{M}\left(\gamma_{0}\right)$ consists of holomorphic planes converging exponentially to $\gamma_{0}$ at $t=\infty$ at $z=\infty$, contains no holomorphic curves with more than one negative ends. Moreover, we have the following result:

Lemma 7.8 The boundary operator $\partial: C_{*}(\alpha) \rightarrow C_{*-1}(\alpha)$ satisfies $\partial \circ \partial=0$, at least when $* \leq 2 m_{0}$. 
Proof Condition 7.1 implies that for $1<m \leq m_{o}$,

$$
\partial \gamma_{j}^{m}=0 \quad \forall j=1,2, \ldots, s,
$$

and hence

$$
\partial^{2} \gamma_{0}^{m}=\partial\left(\sum_{j=1}^{s} a_{j} \gamma_{j}^{m}\right)=0 \quad \forall j=1,2, \ldots, s .
$$

Now consider the $m=1$ case. Observe that $\partial^{2}\left(\gamma_{j}\right)=0$ for $j=1, \ldots, s$ because $C_{-1}(\alpha)=0$. Also $\partial^{2}\left(\gamma_{0}\right)=0$ since $\partial \gamma_{o}=\sum_{j=1}^{s} b_{j} \gamma_{j}$ and $\partial \gamma_{j}=0$ for $j=$ $1, \ldots, s$. Thus $\partial^{2}=0$ at least on $C_{*}(\alpha)$ with $* \leq 2 m_{o}$.

Therefore we can apply to $M$ the stabilization technique as before and obtain the $n=2$ version of Theorem 7.2 ,

Theorem 7.3 Let $(M, \xi)$ be a 3-dimensional subcritical Stein-fillable contact manifold, and $(V, J)$ a subcritical Stein domain such that $\partial V=M$ and $\xi$ is the maximal complex subbundle of $T M$. Then

$$
H C_{i}(M, \xi) \cong \underset{m \in \mathbb{N} \cup\{0\}}{\oplus} H_{2(n+m-1)-i}(V) .
$$

This completes the proof of the Main Theorem.

\section{Acknowledgements}

The author is very grateful to Y Eliashberg for years of guidance, and for many valuable discussion and suggestions. The author is also deeply thankful to S S Kim for reading the draft of this paper, and to the referees for pointing out mistakes and giving many valuable comments and suggestions on an earlier version of this paper.

\section{References}

[1] F Bourgeois, A Morse-Bott approach to contact homology, thesis (2002)

[2] F Bourgeois, Introduction to contact homology, Summer School in Berder: Holomorphic curves and contact topology, (June 2003)

[3] F Bourgeois, Y Eliashberg, H Hofer, K Wysocki, E Zehnder, Compactness result in Symplectic Field Theory, Geom. Topol. 7 (2003) 799-888 MathReview 
[4] Yu W Chekanov, Differential algebras of Legendrian links, Invent. Math. 150 (2002) 441-483 MathReview

[5] K Cieliebak, Y Eliashberg, H Hofer, Symplectic homology of Weinstein manifolds, preprint

[6] C C Conley, E Zehnder, Morse type index theory for flows and periodic solutions of Hamiltonian equations, Comm. Pure Appl. Math. 37 (1984) 283-299 MathReview

[7] Y Eliashberg, Classification of overtwisted contact structures, Invent. Math. 98 (1989) 623-637 MathReview

[8] Y Eliashberg, Topological classification of Stein manifolds of dimension $>2$. Internat. J. Math. 1 (1990) 29-46 MathReview

[9] Y Eliashberg, Symplectic geometry of plurisubharmonic functions with notes by M Abreu, from: "Gauge theory and symplectic geometry (Montreal, PQ, 1995)", NATO Adv. Sci. Inst. Ser. C, Math. Phys. Sci. 488, Kluwer, Dordrecht (1997) 49-67 MathReview

[10] Y Eliashberg, Invariants in contact topology. from: "Proceedings of ICM (Berlin 1998) II", Doc. Math. (1998) Extra Vol. II, 327-338 MathReview

[11] Y Eliashberg, Symplectic topology in the nineties. Symplectic geometry. Differential Geom. Appl. 9 (1998) 59-88 MathReview

[12] Y Eliashberg, A Givental, H Hofer, Introduction to Symplectic Field Theory, from: "GAFA 2000 (Tel Aviv, 1999)", Geom. Funct. Anal. (2000) Special Volume, Part II, 560-673 MathReview

[13] H Geiges, Contact structures on $(n-1)$-connected $(2 n-1)$-manifolds, Pacific J. Math. 161 (1993) 129-137 MathReview

[14] H Geiges, Applications of contact surgery, Topology 36 (1997) 1193-1220 MathReview

[15] E Giroux, Une structure de contact, même tendue, est plus ou moins tordue. Ann. Sci. École Norm. Sup. (4) 27 (1994) 697-705 MathReview

[16] J W Gray, Some global properties of contact structures. Ann. of Math. 69 (1959) 421-450 MathReview

[17] M Gromov, Pseudo-holomorphic curves in symplectic manifolds, Invent. Math. 82 (1985) 307-347 MathReview

[18] H Hofer, K Wysocki, E Zehnder, A characterization of the tight threesphere, Duke Math. J. 81 (1995) 159-226 MathReview

[19] H Hofer, K Wysocki, E Zehnder, A characterization of the tight three-sphere II, Comm. Pure Appl. Math. 52 (1999) 139-1177 MathReview

[20] H Hofer, K Wysocki, E Zehnder, Properties of pseudoholomorphic curves in symplectizations I: Asymptotics, Ann. Inst. Henri Poincaré, 13 (1996) 337-379 MathReview 
[21] H Hofer, K Wysocki, E Zehnder, Properties of pseudo-holomorphic curves in symplectizations III: Fredholm theory, from: "Topics in nonlinear analysis", Progr. Nonlinear Differential Equations Appl. 35, Birkhäuser, Basel (1999) 381475 MathReview

[22] K Honda, On the classification of tight contact structures I: Lens spaces, solid tori and $T^{2} \times I$, Geom. Topol. 4 (2000) 309-368 MathReview

[23] K Honda, On the classification of tight contact structures II: Torus bundles which fiber over the circle, J. Differential Geom. 55 (2000) 83-143 MathReview

[24] P Kronheimer, T Mrowka, Monopoles and contact structures, Invent. Math. 130 (1997) 209-255 MathReview

[25] R Lutz, Structures de contact sur les fibrés principaux en cercles de dimension 3, Ann. Inst. Fourier (Grenoble) 27 (1977) 1-15 MathReview

[26] J Martinet, Formes de contact sur les variétés de dimension 3, from: "Proceedings of Liverpool Singularities Symposium, II (1969/1970)", Lecture Notes in Math. 209, Springer, Berlin (1971) 142-163 MathReview

[27] D McDuff, D Salamon, Introduction to Symplectic Topology, Oxford Mathematical Monographs, Oxford University Press (199 MathReview

[28] D McDuff, D Salamon, J-holomorphic curves and quantum cohomology, University Lecture Series 6, Amer. Math. Soc. (1996) MathReview

[29] J Milnor, Morse Theory, based on lecture notes by M Spivak and R Wells, Annals of Math. Studies 51, Princeton Univ. Press, Princeton, NJ (1963) MathReview

[30] J Robbin, D Salamon, The Maslov index for paths, Topology 32 (1993) 827844 MathReview

[31] D Salamon, E Zehnder, Morse theory for periodic solutions of Hamiltonian systems and the Maslov index, Comm. Pure Appl. Math. 45 (1992) 1303-1360 MathReview

[32] I Ustilovsky, Connected sum construction and contact structures on $S^{4 m+1}$, preprint (1999)

[33] I Ustilovsky, Contact homology and contact structures on $S^{4 m+1}, \mathrm{PhD}$ thesis (1999)

[34] A Weinstein, Contact surgery and symplectic handlebodies, Hokkaido Math. J. 20 (1991) 241-251 MathReview 